\title{
Elucidating conductivity-permselectivity tradeoffs in electrodialysis and reverse electrodialysis by structure-property analysis of ion-exchange membranes
}

\author{
Hanqing Fan ${ }^{\mathrm{a}}$, Ngai Yin Yip ${ }^{\mathrm{a}, \mathrm{b}, *}$ \\ ${ }^{a}$ Department of Earth and Environmental Engineering, Columbia University, New York, NY 10027-6623, United States \\ ${ }^{\mathrm{b}}$ Columbia Water Center, Columbia University, New York, NY 10027-6623, United States
}

\section{A R T I C L E I N F O}

\section{Keywords:}

Ion-exchange membrane

(reverse) electrodialysis

Conductivity-permselectivity tradeoff

Nernst-Planck transport

Counterion condensation theory

\begin{abstract}
A B S T R A C T
Ion-exchange membranes (IEMs) are used in environmental and energy technologies of electrodialysis (ED) desalination and reverse electrodialysis (RED) power generation, respectively. Recent studies reported empirical evidence that the conductivity and permselectivity of IEMs are bound by a tradeoff relationship, where an increase in ionic conductivity is accompanied by a decrease in counterion selectivity over co-ion. A fundamental understanding of this conductivity-permselectivity tradeoff is principal to inform membrane development. This study presents an IEM transport model to analytically relate conductivity and permselectivity to intrinsic membrane chemical and structural properties. The model employs the Nernst-Planck transport framework and incorporates counterion condensation theory to simulate the performance of IEMs in a range of ED and RED operations. The analysis revealed the mechanism for the tradeoff induced by bulk solution concentration: a higher salinity suppresses IEM charge-exclusion, thus lowering permselectivity, but elevates mobile ion concentration within the membrane matrix to improve conductivity. As such, IEM applications are practically confined to sub-seawater salinities, i.e., RED using hypersaline streams will not be efficient. In another tradeoff driven by IEM water sorption, increasing membrane swelling enhances effective ion diffusivity to raise conductivity, but diminishes permselectivity due to dilution of fixed charges. The transport model indicates that increasing membrane ion-exchange capacity and reducing thickness can yield highly selective and conductive IEMs.
\end{abstract}

\section{Introduction}

Ion-exchange membranes (IEMs) are charged polymeric films that allow the selective transport of oppositely-charged species (counterions), while retaining the like-charged ions (co-ions) and water [1]. IEMs are employed in environmental and energy technologies, such as desalination, fuel cells, and salinity gradient power generation [2-5], and also chemical production by the chloralkali process [6]. In electrodialysis (ED) desalination, the application of an electric current drives the separation of ions from saline brackish water, across the IEMs, to produce freshwater $[2,7]$. Reverse electrodialysis (RED), the power generation analog of ED, converts the chemical potential energy stored in salinity gradients to useful electrical work by the directional permeation of ions across the charge-selective membranes [8,9]. In both ED and RED, permeability of the IEM to ions and charge selectivity are principal parameters that determine performance.
A tradeoff relationship between permeability and selectivity exists for membrane processes of gas separation, reverse osmosis, and ultrafiltration [10-14]. Empirical evidence from recent studies indicates IEMs are also bound by a similar constraint - an increase in ionic conductivity (i.e., permeability) is almost inevitably accompanied by a decrease in selectivity for counterions over co-ions, termed permselectivity $[5,15,16]$. Because of this conductivity-permselectivity tradeoff for ion-exchange membranes, any efforts to improve separation will be at the expense of reduced kinetics, thus restricting overall ED and RED performance and energy efficiency [5,15-17]. As such, the tradeoff phenomenon has profound impacts on ED, RED, and other IEM-based processes.

Fundamental understanding of the tradeoff and its intrinsic relation to membrane properties is crucial to inform the development of better membranes. However, while theoretical explanations for the tradeoff of other membrane processes are available [10-14], a complete fundamentals-based

\footnotetext{
* Corresponding author at: Department of Earth and Environmental Engineering, Columbia University, New York, NY 10027-6623, United States.

E-mail address: n.y.yip@columbia.edu (N.Y. Yip).
} 


\begin{tabular}{|c|c|c|c|}
\hline \multicolumn{2}{|c|}{ Nomenclature } & SD & $\begin{array}{l}\text { swelling degree (volume of water sorbed per unit dry } \\
\text { polymer weight) }\end{array}$ \\
\hline \multicolumn{2}{|c|}{ Acronyms } & $T$ & absolute temperature \\
\hline & & $\bar{V}$ & partial molar volume \\
\hline IEM & ion-exchange membrane & $\Delta V^{\mathrm{m}}$ & external membrane potential drop including Donnan po- \\
\hline CEM & cation exchange membrane & & tentials at membrane-solution interface \\
\hline AEM & anion exchange membrane & $\mathrm{Z}$ & ratio of $z_{\mathrm{co}} / z_{\mathrm{ct}}$ \\
\hline ED & electrodialysis & $\alpha$ & permselectivity measured by transport number \\
\hline RED & reverse electrodialysis & $\alpha_{\text {app }}$ & apparent permselectivity measured by OCV \\
\hline $\mathrm{HC}$ & high concentration & $\beta$ & membrane activity correction factor for Nernst-Planck \\
\hline \multirow[t]{2}{*}{ LC } & low concentration & & equation, $1+c_{i}^{\mathrm{m}}\left(d \ln \gamma_{i}^{\mathrm{m}} / d c_{i}^{\mathrm{m}}\right)$ \\
\hline & & $\gamma$ & activity coefficient \\
\hline \multicolumn{2}{|c|}{ Symbols } & $\begin{array}{l}\gamma_{ \pm}^{\mathrm{s}} \\
\varepsilon_{0}\end{array}$ & $\begin{array}{l}\text { salt activity coefficient in bulk solution } \\
\text { vacuum permittivity }\end{array}$ \\
\hline$a$ & activity & $\varepsilon_{\mathrm{r}}$ & solvent dielectric constant \\
\hline$b$ & distance between fixed charges on the polymer chain & $\lambda_{\mathrm{B}}$ & Bjerrum length \\
\hline$c$ & molar concentration & $\bar{\mu}$ & electrochemical potential \\
\hline$c_{\text {fix }}^{\mathrm{m}}$ & fixed charge density of membrane & $\mu$ & chemical potential \\
\hline \multirow[t]{2}{*}{$\bar{c}^{\mathrm{m}}$} & concentration normalized by unit volume of solution & $\mu^{\circ}$ & standard state chemical potential \\
\hline & sorbed by membrane (excluding polymer volume) & $\nu$ & dissociation number \\
\hline$e$ & elementary charge & $\xi$ & dimensionless linear charge density of the polymer \\
\hline$f_{\mathrm{w}}$ & volume fraction of water & $\rho$ & resistivity \\
\hline$k_{\mathrm{B}}$ & Boltzmann constant & $\rho_{\mathrm{p}}$ & mass density of dry polymer \\
\hline$l$ & membrane thickness & $\sigma$ & conductivity \\
\hline$\hat{i}$ & current density & $\tau$ & tortuosity \\
\hline$\hat{i}_{\text {tot }}$ & net current density & $\varphi$ & electric potential \\
\hline$t$ & transport number & $\Delta \varphi_{\text {Donnan }}$ & Donnan potential \\
\hline$z$ & ion valence & $\Delta \varphi^{\mathrm{m}}$ & internal membrane potential drop excluding Donnan po- \\
\hline \multirow[t]{2}{*}{$A$} & function for counterion condensation effect, defined by & & tentials at membrane-solution interface \\
\hline & Eq. $(14 c)$ & $\chi$ & ratio of equivalent mobile co-ion concentration to fixed \\
\hline ASR & area specific resistance & & charge density, $c_{\mathrm{co}}^{\mathrm{m}}\left(\nu_{\mathrm{co}} c_{\mathrm{fix}}^{\mathrm{m}}\right)^{-1}$ \\
\hline ASC & area specific conductance & \multirow{2}{*}{\multicolumn{2}{|c|}{ Superscripts and subscripts }} \\
\hline $\mathrm{CE}$ & current efficiency & & \\
\hline$D$ & diffusion coefficient & & \\
\hline$F$ & Faraday constant & $i$ & mass species \\
\hline IEC & ion-exchange capacity & $\mathrm{s}$ & bulk solution phase \\
\hline$J$ & mass flux & $\mathrm{m}$ & membrane phase \\
\hline K & partition coefficient & $\mathrm{ct}$ & counterion \\
\hline OCV & open-circuit voltage & co & co-ion \\
\hline$P$ & hydrostatic pressure & $\mathrm{w}$ & water \\
\hline$R_{\mathrm{g}}$ & gas constant & $\mathrm{P}$ & polymer \\
\hline
\end{tabular}

framework to describe the IEM conductivity-permselectivity relationship is lacking. Although recent experimental studies demonstrated that membrane water uptake and bulk solution concentrations can induce the tradeoff between IEM conductivity and permselectivity, the qualitative explanations provided do not give a rigorous mechanistic explanation of the phenomenon $[15,18]$. Analytical approaches to describe the effects of membrane property on IEM transport are almost always focused on quantifying isolated property-performance relationships [19-21] (such as, ionic conductivity dependence on bulk concentrations) and oftentimes adopt semiempirical models, i.e., employ fitting parameters [18,19,22,23]. Hence, such approaches are unable to elucidate the intrinsic relationship between membrane properties and the intertwined performance parameters of permselectivity and conductivity. The Nernst-Planck framework is commonly used to describe IEM transport [21,24-27], but requires the activity and diffusion coefficient of ions within the charged IEM matrix, which are anticipated to deviate significantly from bulk solution due to non-idealities and are also not readily accessible by experiments [24,25]. Recently, studies showed that counterion condensation theory, originally established for aqueous polyelectrolyte solutions, can be applied to IEM to predict ion activity and diffusivity with reasonable accuracy $[28,29]$, thus highlighting the potential to overcome the present limitations of Nernst-Planck transport models.
This study analyzes ion transport across IEMs to elucidate the dependence of key performance parameters, ionic conductivity and charge selectivity, on intrinsic membrane chemical and structural properties. Firstly, the working principles of ion-exchange membranes, electrodialysis, and reverse electrodialysis are described. The IEM model is presented and principal governing equations are discussed. In the model, ion transport is driven by chemical and electrostatic potential, i.e., the Nernst-Planck equation, and counterion condensation theory is incorporated to account for ion activity and diffusivity within the membrane matrix. Computational codes numerically solve for the system of nonlinear differential equations. After the model is validated with literature data, the effects of operating parameters, electric potential difference and external solution concentrations, on current efficiency and resistance to ion fluxes are examined. The analysis then assessed the dependence of permselectivity and conductivity in ED and RED on the intrinsic membrane properties of ion-exchange capacity, degree of swelling, and membrane thickness. The underpinning phenomena relating operating conditions and membrane properties to IEM performance are highlighted and the principal factors governing the observed conductivity-permselectivity tradeoff are elucidated. Lastly, the implications for ED desalination, RED salinity gradient power generation, and membrane development are discussed. 


\section{Working principles of ion-exchange membranes and IEM processes}

\subsection{Working principles of ion-exchange membranes}

Ion-exchange membranes are water-swollen polymeric films of typically $50-200 \mu \mathrm{m}$ thickness, with a high density of charged ionic functional groups fixed to the backbone chains $[1,30,31]$. The selective transport of IEMs is achieved by the charge exclusion principle: the fixed functional groups exclude like-charged co-ions and the membrane preserves electroneutrality by having a high concentration of counterions (opposite charge to fixed moieties). Ion transport is driven by electrochemical potential, and the comparatively greater concentration results in a larger flux of counterions than co-ions. Hence, IEMs allow the preferential permeation of counterions over co-ions, but because coions are not completely excluded from the membrane matrix, the charge selectivity of IEMs is imperfect. Cation exchange membranes (CEMs) with fixed negatively charged functional groups, such as, sulfonic acid, phosphoric acid, and derivatives of sulfonamide and azole, selectively favor cations permeation; whereas anion exchange membranes (AEMs) possess cationic functional groups, e.g., quaternary ammonium, to facilitate transport of anions over cations [32,33].

\subsection{Electrodialysis desalination}

Electrodialysis (ED) is an IEM-based desalination technology that utilizes an electric current to separate charged ions from a saline stream and produce freshwater [2,7]. In ED, an external electric potential is applied across a stack comprising repeating pairs of CEM and AEM. Saline feed stream flows through each compartment channel between the membranes. Fig. 1A shows one CEM-AEM pair of the stack and the adjacent solution compartments. The external electric potential drives the permeation of cations towards the cathode and the anions towards the anode. As the IEMs selectively allow the permeation of counterions and retain co-ions, cations and anions permeate into the concentrate compartment from the abutting diluate streams, across the CEM and AEM, respectively, but ions in the concentrate stream are hindered from crossing into the diluate compartment (Fig. 1A). This selectivity for current carriers generates alternating diluted and concentrated flows across the stack. For converting the ionic current to an electric current, a reversible redox couple (e.g., $\mathrm{Fe}^{2+} / \mathrm{Fe}^{3+}$ or $\left.\mathrm{Fe}(\mathrm{CN})_{6}\right]^{4-} /[\mathrm{Fe}$ $\left.(\mathrm{CN})_{6}\right]^{3-}$ ) is circulated between the end electrodes, thereby closing the circuit $[2,7]$.

\subsection{Reverse electrodialysis energy production}

Reverse electrodialysis (RED) produces useful work from salinity gradients by inverting the operating principles of ED separation [9,34]. Instead of supplying external electrical energy, RED generates power from the controlled mixing of high and low concentration solutions. A similar membrane stack configuration as ED is utilized (Fig. 1B): a repeating cell consists of, in spatial order, a CEM, a high concentration (HC) solution compartment, an AEM, and a low concentration (LC) solution compartment (which is bordered by the CEM of the next cell). This alternating structure enables the series addition of Nernst potentials arising from the concentration difference across the IEMs. Selective transport of anions and cations in the HC solution, across the AEM and CEM, respectively, to the adjacent LC chambers produces a net charged ion flux. Like ED, a reversible redox couple is circulated between the two end electrode chambers to transform ionic current in the stack to an electric current that powers the load in the external circuit.

\section{Transport model for ion-exchange membranes}

In this section, the transport model for ion permeation in IEMs is presented and key equations are listed. The model considers the solution-membrane interface to be at quasi electrochemical equilibrium and uses the modified Nernst-Planck equation to describe ion fluxes within the membrane matrix under an electrochemical potential gradient. Manning's counterion condensation model is utilized to account for ion activities and diffusion coefficients within the membrane matrix, which differ significantly from bulk solution. Membrane performance parameters in ED and RED are then introduced and their significance is discussed.

\subsection{Equilibrium concentrations at solution-membrane interface}

Fig. 2A shows an IEM of homogenous fixed charge density, $c_{\mathrm{fix}}^{\mathrm{m}}$ (dotted horizontal green line), separating high and low concentration solutions of $c^{s, \mathrm{HC}}$ and $c^{s, \mathrm{LC}}$, respectively. At equilibrium at the solutionmembrane interfaces, the electrochemical potential, $\bar{\mu}_{i}$, of species $i=$ counterions, co-ions, (or water) in solution and membrane are equal:

$\bar{\mu}_{i}^{\mathrm{m}}=\bar{\mu}_{i}^{\mathrm{s}}$

where superscripts $\mathrm{m}$ and $\mathrm{s}$ denote membrane and solution phase, respectively. The electrochemical potential is the sum of the chemical potential, $\mu_{i}$, and the electrical potential, $\bar{\mu}_{i}=\mu_{i}+z_{i} F \varphi$ ( $z$ is the valency of the ion, $F$ is the Faraday constant, and $\varphi$ is the local electrostatic potential at the solution-membrane interface) [35]. Chemical potential, $\mu_{i}$, can be further expressed by the standard state chemical potential, $\mu_{i}^{0}$, activity of species, $a_{i}$, and hydrostatic pressure, $P$ : $\mu_{i}=\mu_{i}^{0}+R_{\mathrm{g}} T \ln a_{i}+\bar{V}_{i} P[36]$. Here, $R_{\mathrm{g}}$ is the gas constant, $T$ is the absolute temperature, and $\bar{V}$ is the partial molar volume of $i$. Thus, the electrochemical potential of species $i$ is

$\bar{\mu}_{i}=\mu_{i}^{0}+R_{\mathrm{g}} T \ln a_{i}+\bar{V}_{i} P+z_{i} F \varphi$

As both ED and RED operate at practically ambient hydraulic pressures, the hydrostatic pressure difference between the membrane
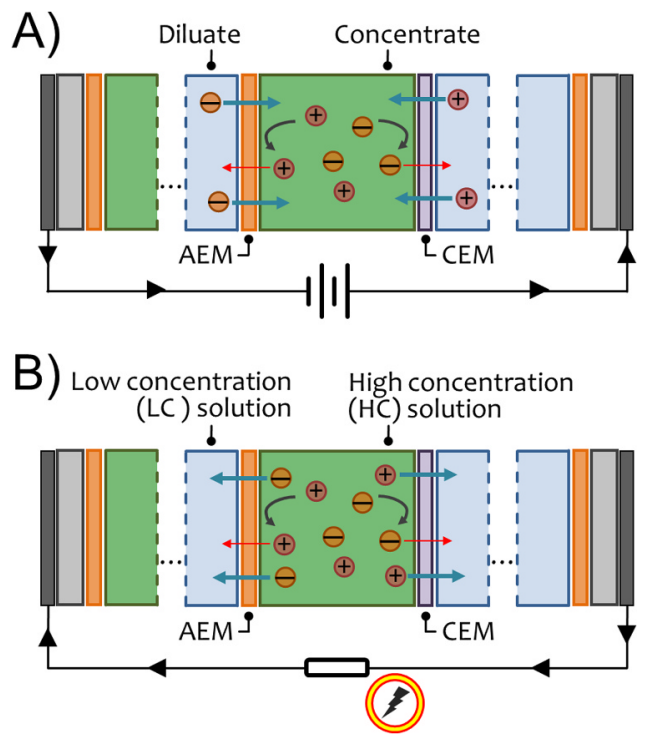

Fig. 1. Schematic of A) electrodialysis (ED) and B) reverse electrodialysis (RED). The arrows indicate direction of cation $(+)$ and anion $(-)$ permeation. Repeating pairs of negatively charged cation exchange membranes (CEM) and positively charged anion exchange membranes (AEM) allow the selective permeation of oppositely charged counterions (cations and anions, respectively), while rejecting co-ions. Only one IEM pair and the end electrodes are shown. A reversible redox couple is circulated at the end electrodes to convert between the ionic and electric current. In ED, ion separation is achieved by applying an external voltage to drive the permeation of ions against the concentration gradient, i.e., from the diluate to concentrate stream. Conversely in RED, the concentration gradient across the IEM results in a Nernst potential that drives the transport of ions. The net ion flux is transformed to an electric current and powers a load in the external circuit, thereby producing useful work. 


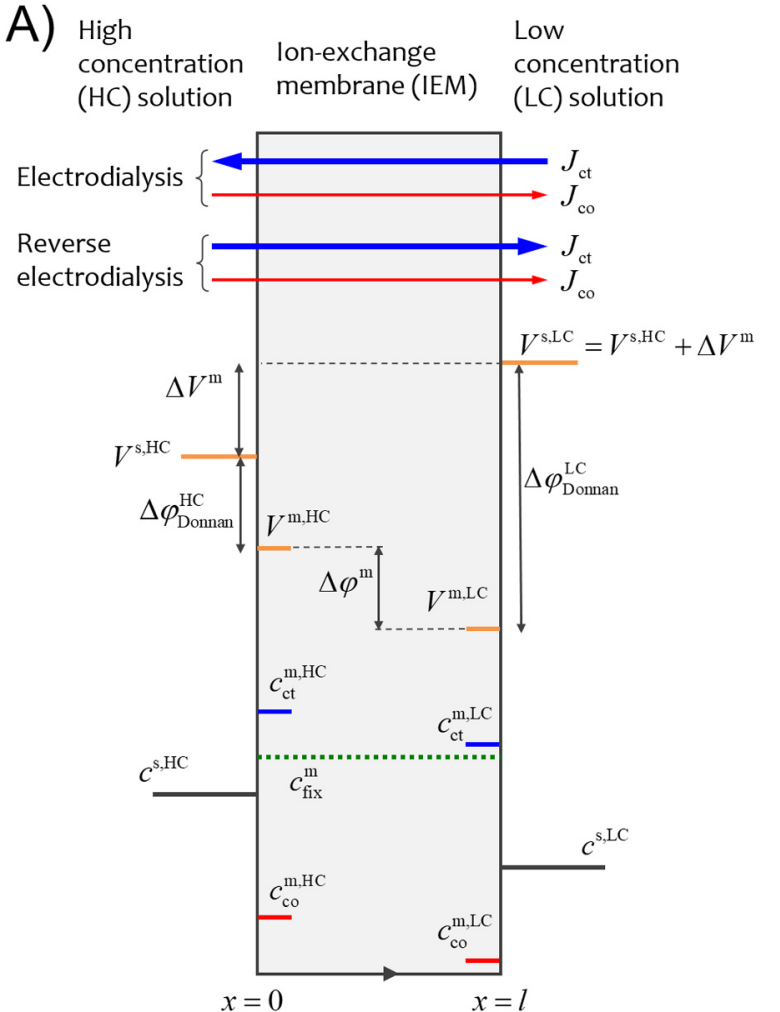

B)

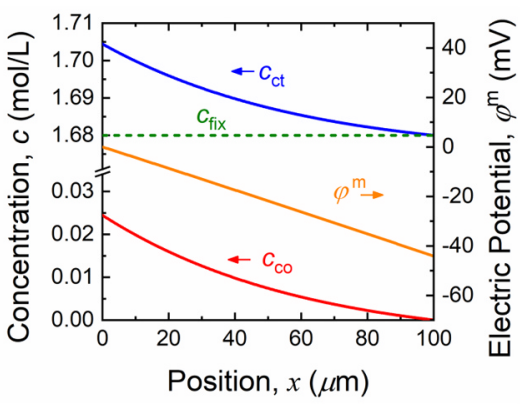

Fig. 2. A) Schematic depicting counter- and co-ion fluxes, $J_{\mathrm{ct}}$ and $J_{\mathrm{co}}$, respectively, in ED and RED. Also indicated are representative bulk, counterion, and co-ion concentrations at the solution-membrane interfaces, $c^{\mathrm{s}}, c_{\mathrm{ct}}^{\mathrm{m}}$, and $c_{\mathrm{co}}^{\mathrm{m}}$, respectively, and uniform fixed charge density, $c_{\text {fix }}^{\mathrm{m}}$, across the membrane. Electric potentials, $V$, at the solution and membrane sides of the HC and LC interfaces are denoted (superscripts s, m, HC, and LC) for an illustrative RED process. The external and internal electric potential difference across the IEM, $\Delta V^{\mathrm{m}}$ and $\Delta \varphi^{\mathrm{m}}$, are related through the Donnan potentials of the solution-membrane interfaces, $\Delta \varphi_{\text {Donnan. }}$ B) Concentration profiles of counterion, co-ion, and fixed charge within the IEM for an illustrative RED process (blue solid, red solid, and green dotted lines, respectively). Electric potential profile within the membrane is depicted by orange line. For the RED example shown here, $\Delta \varphi^{\mathrm{m}}$ is $<0$, but is positive for ED. Membrane properties $c_{\mathrm{fix}}=1.68 \mathrm{eq} / \mathrm{L}, f_{\mathrm{w}}=0.30$, and $l$ $=100 \mu \mathrm{m}$; bulk solutions $c^{\mathrm{s}, \mathrm{HC}}$ and $c^{\mathrm{s}, \mathrm{LC}}$ are 600 and $17 \mathrm{mM} \mathrm{NaCl}$, respectively.

and solution phases can be approximated to zero: $P^{\mathrm{m}}-P^{\mathrm{s}}=0$. By selecting the same standard state chemical potential for both phases, $\mu_{i}^{m, 0}$ $=\mu_{i}^{s, 0}[24]$, Eqs. (1) and (2) combined reduces to just the activity and electrostatic terms:

$R_{\mathrm{g}} T \ln a_{i}^{m}+z_{i} F \varphi^{\mathrm{m}}=R_{\mathrm{g}} T \ln a_{i}^{s}+z_{i} F \varphi^{\mathrm{s}}$

Activity, $a$, can be replaced with the product of the concentration, $c$, and activity coefficient, $\gamma$, which is a function of concentration. Here, concentration is moles of solute per unit volume of solution. However, the membrane matrix comprises both solution and polymer.
Multiplying $\bar{c}^{\mathrm{m}}$, the concentration normalized by unit volume of solution sorbed in the membrane matrix (i.e., excluding the polymer volume) by the volume fraction of water in the IEM, $f_{\mathrm{w}}$, gives the concentration normalized by total volume of the water-swollen membrane, $c^{\mathrm{m}}=f_{\mathrm{w}} \bar{c}^{\mathrm{m}}$ (i.e., includes volume of solution and polymer). For the coions and counterions (subscripts co and ct, respectively), Eq. (3) can be correspondingly rearranged to

$\frac{\gamma_{\mathrm{co}}^{m} c_{\mathrm{co}}^{m}}{f_{\mathrm{w}} \gamma_{\mathrm{co}}^{s} c_{\mathrm{co}}^{s}}=\exp \left[-\frac{z_{\mathrm{co}} F}{R_{\mathrm{g}} T} \Delta \varphi_{\text {Donnan }}\right]$

$\frac{\gamma_{\mathrm{ct}}^{m} c_{\mathrm{ct}}^{m}}{f_{\mathrm{w}} \gamma_{\mathrm{ct}}^{s} c_{\mathrm{ct}}^{s}}=\exp \left[-\frac{z_{\mathrm{ct}} F}{R_{\mathrm{g}} T} \Delta \varphi_{\text {Donnan }}\right]$

The difference in electrostatic potential across the solution-membrane interface, $\varphi^{\mathrm{m}}-\varphi^{\mathrm{s}}$, is defined as the Donnan potential, $\Delta \varphi$ Donnan $[1,37,38]$.

Equating the Donnan potentials of Eqs. (4a) and (4b) yields Eq. (5) after reordering:

$c_{\mathrm{co}}^{m}=f_{\mathrm{w}}{ }^{1-Z}\left(\gamma_{\mathrm{ct}}^{s} c_{\mathrm{ct}}^{s}\right)^{-Z} \gamma_{\mathrm{co}}^{s} c_{\mathrm{co}}^{s}\left(\gamma_{\mathrm{co}}^{m}\right)^{-1}\left(\gamma_{\mathrm{ct}}^{m} c_{\mathrm{ct}}^{m}\right)^{Z}$

where $\mathrm{Z}$ is $z_{\mathrm{co}} / z_{\mathrm{ct}}$. Counter- and co-ion activity coefficients within the membrane, $\gamma_{\mathrm{ct}}^{m}$ and $\gamma_{\mathrm{co}}^{m}$, respectively, can be predicted using Manning's counterion condensation model with good agreement with experimental data $[28,39]$ :

$\gamma_{\mathrm{co}}^{m}=\exp \left\{-\frac{1}{2} Z^{2}\left[1+\nu_{\mathrm{ct}}\left|z_{\mathrm{ct}}\right| \xi\left(\nu_{\mathrm{co}}+\nu_{\mathrm{ct}}\right) \chi\right]^{-1}\right\}$

$\gamma_{\mathrm{ct}}^{m}=\frac{\left|z_{\mathrm{ct}}\right|^{-1} \xi^{-1}+\nu_{\mathrm{ct}}\left|z_{\mathrm{ct}}\right| \chi}{1+\nu_{\mathrm{ct}}\left|z_{\mathrm{ct}}\right| \chi} \exp \left\{-\frac{1}{2}\left[1+v_{\mathrm{ct}}\left|z_{\mathrm{ct}}\right| \xi\left(\nu_{\mathrm{co}}+v_{\mathrm{ct}}\right) \chi\right]^{-1}\right\}$

with $\chi=c_{\mathrm{co}}^{\mathrm{m}}\left(\nu_{\mathrm{co}} c_{\mathrm{fix}}^{\mathrm{m}}\right)^{-1}$ and $\xi$ being the dimensionless linear charge density of the polymer:

$\xi=\frac{\lambda_{\mathrm{B}}}{b}=\frac{e^{2}}{4 \pi \varepsilon_{0} \varepsilon_{r} k_{\mathrm{B}} T b}$

where $\lambda_{\mathrm{B}}$ is the Bjerrum length, $b$ is the distance between fixed charges on the polymer chain, $e$ is the elementary charge, $\varepsilon_{0}$ is the vacuum permittivity, $\varepsilon_{\mathrm{r}}$ is the solvent dielectric constant, and $k_{\mathrm{B}}$ is the Boltzmann constant. Eqs. (6a) and (6b) are applicable for $\xi>\left|z_{\mathrm{ct}}\right|^{-1}$; this condition is readily satisfied for the high density of fixed charges within an IEM [40].

For 1:1 electrolytes, such as $\mathrm{NaCl}$ and $\mathrm{MgSO}_{4}, z_{\mathrm{co}}=-z_{\mathrm{ct}}$ and, hence, $\mathrm{Z}$ is -1 . Additionally substituting in $c_{\mathrm{co}}^{\mathrm{s}}=\nu_{\mathrm{co}} c^{\mathrm{s}}, c_{\mathrm{ct}}^{\mathrm{s}}=\nu_{\mathrm{ct}} c^{\mathrm{s}}$, and $\gamma_{\mathrm{co}}^{\mathrm{s}} \gamma_{\mathrm{ct}}^{\mathrm{s}}=\left(\gamma_{ \pm}^{\mathrm{s}}\right)^{2}$ [41] further reduces Eq. (5) to

$c_{\mathrm{co}}^{m}=\frac{\nu_{\mathrm{co}} \nu_{\mathrm{ct}} f_{\mathrm{w}}^{2}\left(\gamma_{ \pm}^{\mathrm{s}} c^{s}\right)^{2}}{\gamma_{\mathrm{co}}^{m} \gamma_{\mathrm{ct}}^{m} c_{\mathrm{ct}}^{m}}$

where $\nu$ is the number of counter- or co-ions each electrolyte molecule dissociates into (i.e., $\nu_{\mathrm{co}}=\nu_{\mathrm{ct}}=1$ for $\mathrm{NaCl}$ ). The salt activity coefficient in bulk solution, $\gamma_{+}^{\mathrm{s}}$, can be predicted using theoretical models; in this study the Pitzer equations are employed for $\mathrm{NaCl}[42,43]$. Eqs. (1)-(5) are general equations for all electrolytes, whereas Eq. (7) is specific to 1:1 electrolytes. The equations are applicable for equilibrium at both HC and LC solution-membrane interfaces (Fig. 2A).

Besides the condition of electrochemical potential equilibrium between the solution and membrane phases, charge balance also needs to be preserved at all points within the membrane, including the interfaces:

$\left|z_{\mathrm{co}}\right| c_{\mathrm{co}}^{m}+c_{\mathrm{fix}}=\left|z_{\mathrm{ct}}\right| c_{\mathrm{ct}}^{m}$

Rearranging Eq. (8) yields

$c_{\mathrm{co}}^{m}=\frac{\left|z_{\mathrm{ct}}\right| c_{\mathrm{ct}}^{m}-c_{\mathrm{fix}}}{\left|z_{\mathrm{co}}\right|}$

which can be solved simultaneously with Eq. (5) (general case) or 7 (1:1 
electrolytes) to determine $c_{\mathrm{co}}^{\mathrm{m}}$ and $c_{\mathrm{ct}}^{\mathrm{m}}$. The counter- and co-ion concentrations at the HC and LC solution-membrane interfaces are indicated in Fig. $2 \mathrm{~A}$ as $c_{\mathrm{ct}}^{m, \mathrm{HC}}, c_{\mathrm{co}}^{m, \mathrm{HC}}, c_{\mathrm{ct}}^{m, \mathrm{LC}}$, and $c_{\mathrm{co}}^{m, \mathrm{LC}}$, respectively. The Donnan potential, $\Delta \varphi_{\text {Donnan }}=\varphi^{\mathrm{m}}-\varphi^{\mathrm{s}}$, is calculated using Eq. (4) with the membrane and solution activities, $\gamma_{i}^{\mathrm{m}} c_{i}^{\mathrm{m}}$ and $\gamma_{i}^{\mathrm{s}} c_{i}^{\mathrm{s}}$, and approximating the activity coefficient of counter- and co-ions in bulk solution to be effectively the same, i.e., $\gamma_{\mathrm{ct}}^{\mathrm{s}}=\gamma_{\mathrm{co}}^{\mathrm{s}}=\gamma_{+}^{\mathrm{s}}$. Indicated in Fig. $2 \mathrm{~A}$ are $\varphi^{\mathrm{s}}, \varphi^{\mathrm{m}}$, and $\Delta \varphi_{\text {Donnan }}$ for the HC and LC solution-membrane interfaces. For this study, $\mathrm{NaCl}$, a 1:1 electrolyte, is employed. The effect of $\mathrm{pH}$ is negligible, since the contribution of $\mathrm{H}^{+}$and $\mathrm{OH}^{-}$to the electrolyte composition is small $\left(<10^{-4} \mathrm{M}\right)$ compared to $\mathrm{Na}^{+}$and $\mathrm{Cl}^{-}$concentrations $\left(>10^{-2} \mathrm{M}\right)$ across the environmentally-relevant $\mathrm{pHs}$ of 4-10.

\subsection{Nernst-Planck equation for transport across membrane}

During electrodialysis separation or reverse electrodialysis energy production, a net driving force is present for transport of species across the ion-exchange membrane. At steady state, the molar ion flux is governed by [44]:

$J_{i}=-\frac{D_{i}^{\mathrm{m}} c_{i}^{\mathrm{m}}}{R_{\mathrm{g}} T} \frac{d \bar{\mu}_{i}^{\mathrm{m}}}{d x}$

where $D^{\mathrm{m}}$ is the ion diffusion coefficient and $x$ is the flux direction, i.e., across membrane thickness. The electrochemical potential of counteror co-ions in the membrane, $\bar{\mu}_{i}^{\mathrm{m}}$, is described by Eq. (2) and includes the electrical potential and activity terms, $z_{\mathrm{i}} F \varphi+R_{\mathrm{g}} T \ln \gamma c$, assuming no hydraulic pressure gradient within the membrane matrix. Substituting the terms into Eq. (10) yields the Nernst-Planck equation (modified to account for activity coefficient):

$J_{i}=-D_{i}^{\mathrm{m}} c_{i}^{\mathrm{m}}\left(\frac{d \ln \left(\gamma_{i}^{\mathrm{m}} c_{i}^{\mathrm{m}}\right)}{d x}+\frac{z_{i} F}{R_{\mathrm{g}} T} \frac{d \varphi^{\mathrm{m}}}{d x}\right)$

The first term of Eq. (11) denotes Fickian diffusion of ions down an activity or, effectively, concentration gradient whereas the second term signifies migration of the charged species under an electric field within the membrane. Expressions for the local concentration and electric potential gradients within the membrane can be derived by combining Eq. (11) for counter- and co-ions:

$\frac{d c^{\mathrm{m}}}{d x}=\left(\frac{J_{\mathrm{co}}}{z_{\mathrm{co}} D_{\mathrm{co}}^{\mathrm{m}} c_{\mathrm{co}}^{\mathrm{m}}}-\frac{J_{\mathrm{ct}}}{z_{\mathrm{ct}} D_{\mathrm{ct}}^{\mathrm{m}} c_{\mathrm{ct}}^{\mathrm{m}}}\right)\left(\frac{\beta_{\mathrm{ct}}}{z_{\mathrm{ct}} c_{\mathrm{ct}}^{\mathrm{m}}}-\frac{\beta_{\mathrm{co}}}{z_{\mathrm{co}} c_{\mathrm{co}}^{\mathrm{m}}}\right)^{-1}$

$\frac{d \varphi^{\mathrm{m}}}{d x}=\frac{R_{\mathrm{g}} T}{F}\left(\frac{J_{\mathrm{co}}}{D_{\mathrm{co}}^{\mathrm{m}} \beta_{\mathrm{co}}^{\mathrm{m}}}-\frac{J_{\mathrm{ct}}}{D_{\mathrm{ct}}^{\mathrm{m}} \beta_{\mathrm{ct}}^{\mathrm{m}}}\right)\left(\frac{z_{\mathrm{ct}} c_{\mathrm{ct}}^{\mathrm{m}}}{\beta_{\mathrm{ct}}}-\frac{z_{\mathrm{co}} c_{\mathrm{co}}^{\mathrm{m}}}{\beta_{\mathrm{co}}}\right)^{-1}$

where $\beta_{i}=1+c_{i}^{\mathrm{m}}\left(d \ln \gamma_{i}^{\mathrm{m}} / d c_{i}^{\mathrm{m}}\right)$. The concentration and electric potential gradients are functions of the ion fluxes and local membrane concentrations. It is instructive to note that, because of the electroneutrality constraint, the slopes of counter- and co-ion concentrations are identical and $d c^{\mathrm{m}} / d x$ of Eq. (12) is applicable to both $c_{\mathrm{ct}}$ and $c_{\mathrm{co}}$. Summing the two Donnan potentials at the solution-membrane interfaces and the internal electric potential difference within the IEM, $\Delta \varphi^{\mathrm{m}}$, yields the external electric potential difference across the membrane, $\Delta V^{\mathrm{m}}=\Delta \varphi^{\mathrm{m}}+\Delta \varphi_{\text {Donnan }}^{\mathrm{HC}}-\Delta \varphi_{\text {Donnan }}^{\mathrm{LC}}$ (Fig. 2A).

Numerical solutions for $J_{\text {co }}$ and $J_{\text {ct }}$ can be obtained using the finite difference method: the membrane thickness is discretized into one-dimensional finite elements, across which Eqs. 12 and 13 are simultaneously solved to satisfy both charge balance along the entire membrane thickness (i.e., local electroneutrality, Eq. (8)) and boundary conditions determined in the preceding section, i.e., $c_{i}^{\mathrm{m}}=c_{i}^{m, \mathrm{HC}}$ and $\varphi^{\mathrm{m}}=\varphi^{m, \mathrm{HC}}$ at $x=0$, and $c_{i}^{\mathrm{m}}=c_{i}^{m, \mathrm{LC}}$ and $\varphi^{\mathrm{m}}=\varphi^{m, \mathrm{LC}}$ at $x=l$. Python codes are used to converge on the pair of ion fluxes that meets all constraints: the interfacial ion concentrations, $c_{i}^{m, \mathrm{HC}}$ and $c_{i}^{m, \mathrm{LC}}$ (which are at equilibrium with external bulk concentrations) and the $\Delta V^{\mathrm{m}}$ specified (or the equivalent $\Delta \varphi^{\mathrm{m}}$ ).

Effective ion diffusivities within the membrane matrix of polymer and sorbed solution account for the lengthened diffusional pathway, i.e., increased tortuosity and fraction of free volume (equivalently, water volume fraction, $f_{\mathrm{w}}$ ) $<1$, and are modeled using the lattice approach by multiplying the diffusion coefficient in free space with the factor $f_{\mathrm{w}}{ }^{2} /\left(2-f_{\mathrm{w}}\right)^{2}[45,46]$. The diffusion coefficient of counter- and co-ions under the locally inhomogeneous electric field of a charged ionexchange membrane can be predicted using the counterion condensation model $[29,39]$. Incorporating the tortuosity factor and fraction of free volume, $D_{\mathrm{co}}^{\mathrm{m}}$ and $D_{\mathrm{ct}}^{\mathrm{m}}$ can be described by

$D_{\mathrm{co}}^{m}=D_{\mathrm{co}}^{s}\left(\frac{f_{\mathrm{w}}}{2-f_{\mathrm{w}}}\right)^{2}\left(1-\frac{\left|z_{\mathrm{co}}\right|^{2} A}{3}\right)$

$D_{\mathrm{ct}}^{m}=D_{\mathrm{ct}}^{s}\left(\frac{f_{\mathrm{w}}}{2-f_{\mathrm{w}}}\right)^{2}\left(\frac{\left|z_{\mathrm{ct}}\right|^{-1} \xi^{-1}+\nu_{\mathrm{ct}}\left|z_{\mathrm{ct}}\right| \chi}{1+v_{\mathrm{ct}}\left|z_{\mathrm{ct}}\right| \chi}\right)\left(1-\frac{\left|z_{\mathrm{ct}}\right|^{2} A}{3}\right)$

$A=\left.\left|z_{\mathrm{ct}}\right|^{-2} \sum_{m_{1}=-\infty}^{\infty} \sum_{m_{2}=-\infty}^{\infty}\left[1+\pi\left(m_{1}^{2}+m_{2}^{2}\right)+\left(v_{\mathrm{co}}+v_{\mathrm{ct}}\right)\left|z_{\mathrm{co}} z_{\mathrm{ct}}\right| \xi \chi\right]^{-2}\right|_{\left(m_{1}, m_{2}\right) \neq(0,0)}$

$A \approx\left|z_{\mathrm{ct}}\right|^{-2}\left[1+\pi+\left(\nu_{\mathrm{co}}+\nu_{\mathrm{ct}}\right)\left|z_{\mathrm{co}} z_{\mathrm{ct}}\right| \xi \chi\right]^{-1}$

Eqs. (14a)-(14c) are applicable for $\xi>\left|z_{\mathrm{ct}}\right|^{-1}$. Note that in ED, the counterion flux, $J_{\mathrm{ct}}$, is against the concentration gradient and co-ion flux, $J_{\text {co }}$, is in the opposite direction, whereas both $J_{\text {ct }}$ and $J_{\text {co }}$ are down $d c / d x$ for RED (Fig. 2A). Additionally, the flux of water can be neglected as it is relatively small compared to the ion fluxes [1].

The transport model presented here bears analogous similarity to the solution-diffusion transport mechanism across dense nonporous membranes $[47,48]$, as mentioned in a recent study [29]. In the solution-diffusion model, species partition or sorb from the bulk solution into the membrane at the interface and $c_{i}^{\mathrm{m}} / c_{i}^{\mathrm{s}}=K_{i}$ where $K_{\mathrm{i}}$ is the partition coefficient. Correspondingly, in IEM transport, the distribution of ion concentrations in the solution and membrane at the interface is governed by the Donnan potential, Eqs. (4a) and (4b), and the equivalent partition coefficient is $K_{i}=f_{\mathrm{w}} \gamma_{i}^{s} \exp \left[-z_{i} F \Delta \varphi_{\text {Donnan }} / R_{\mathrm{g}} T\right] / \gamma_{i}^{m}$. Species diffuse across the dense membrane driven by a chemical potential gradient in the solution-diffusion mechanism. This is paralleled by species transport under an electrochemical potential across the ionexchange membrane in ED and RED.

\subsection{Performance parameters for ED and RED}

\subsubsection{Current efficiency}

Current efficiency, CE, is a measure of ionic current utilization in ED and RED for separation and energy production, respectively:

$\begin{aligned} \mathrm{CE}_{\mathrm{ED}} & =\frac{z_{\mathrm{ct}} J_{\mathrm{ct}}}{z_{\mathrm{co}} J_{\mathrm{co}}+z_{\mathrm{ct}} J_{\mathrm{ct}}} \\ \mathrm{CE}_{\mathrm{RED}} & =\frac{z_{\mathrm{co}} J_{\mathrm{co}}+z_{\mathrm{ct}} J_{\mathrm{ct}}}{z_{\mathrm{ct}} J_{\mathrm{ct}}}\end{aligned}$

where $z$ is the ion valence, $J$ is the ion flux, and subscripts ct and co denote counter- and co-ions, respectively. Note that $z$ and $J$ can be positive or negative, depending on charge and direction, and the product $z J$ gives the ionic current. In ED desalination, the electric current drives counter- and co-ion fluxes. These two ion fluxes flow in opposite directions and are of different charge (Fig. 1A), with only the counterion flux performing the desired function of desalinating the saline feed, whereas co-ion flux is an unwanted leakage of ions to the diluate stream that actually compromise desalination performance. Current efficiency for ED desalination is, thus, the ratio of the current due to counterion flux to the total ionic current, Eq. (15a). Conversely, the aim of RED is to generate an ion flux that can then be used to drive an external circuit and, hence, $\mathrm{CE}_{\mathrm{RED}}$ is defined differently from $\mathrm{ED}$ 
desalination. Because both fluxes are in the same direction (Fig. 1B), the current from counterion flux is partly negated by co-ion flux. The RED current efficiency, Eq. (15b), is the net ionic current divided by the current due to counterion flux. Note that equation for $\mathrm{CE}_{\mathrm{RED}}$ is the reciprocal of $C E_{\mathrm{ED}}$. Current efficiency of ED and RED is analogous to the Faradaic efficiency of electrochemical processes, which quantifies the percentage of charge utilized for the desired electrochemical reaction.

\subsubsection{Permselectivity}

Permselectivity, $\alpha$, describes the selectivity for counterion transport and is defined as the ionic current carried by counterion flux less the current from co-ion flux, normalized by the total ionic current $[1,31]$ :

$\alpha=\frac{\left|z_{\mathrm{ct}} J_{\mathrm{ct}}\right|-\left|z_{\mathrm{co}} J_{\mathrm{co}}\right|}{\sum\left|z_{i} J_{i}\right|}=t_{\mathrm{ct}}-t_{\mathrm{co}}$

Note that the sign conventions of $z$ and $J$ are neglected and only the magnitude of the ionic currents are used to calculate $\alpha$. Further, the fraction of total ionic current carried by species $i$ is the transport number, $t_{i}$, and, hence, permselectivity is also the counterion transport number less the co-ion transport number, Eq. (16)[1]. An IEM with perfect charge selectivity is only permeable to counterions but not coions and, therefore, permselectivity equals to one.

Experimentally characterized $\alpha$ measurements are commonly reported in literature, but those values are more accurately termed "apparent permselectivity", $\alpha_{\text {app }}$, and is the ratio of measured open-circuit voltage (OCV) to theoretical Nernst potential [1]. Because of the ease of experimental characterization (one electrochemical reading instead of tracking counter- and co-ion concentration changes), apparent permselectivity is often used as a proxy parameter to approximate the fraction of ionic current carried by counter- and co-ions during actual ED and RED operation even though it deviates from the definition of Eq. (16). Comparison between apparent and real permselectivities are discussed later in Section 5.

\subsubsection{Area specific resistance}

Area specific resistance, ASR, is defined in Eq. (17) as the slope of $\Delta V^{\mathrm{m}}$ with respect to the net current density, $\hat{i}_{\mathrm{tot}}=F\left(z_{\mathrm{co}} J_{\mathrm{co}}+z_{\mathrm{ct}} J_{\mathrm{ct}}\right)$, i.e., differential resistance:

$\operatorname{ASR}=\frac{\mathrm{d} \Delta V^{m}}{\mathrm{~d} \hat{i}_{\text {tot }}}$

Because the contribution of IEM to total internal resistance is significant in ED and RED [17], the membrane ASR should be small to suppress undesired resistive losses. In IEM processes, steady state current-voltage response can be described by one of the three regimes: ohmic (or underlimiting), plateau (or limiting), and overlimiting [49]. The current analysis will focus on simulating ion-exchange membranes working within the ohmic regime, which is the common operating conditions for ED and RED. In this relatively low current regime, i.e., under-limiting the relation between current density and imposed voltage is linear; ion depletion in the concentration polarization boundary layer is not dominant and a limiting current is not reached (i.e., before plateau regime).

\subsubsection{Conductivity}

Conductivity, $\sigma$, is the reciprocal of resistivity, $\rho$, and describes the ability of the IEM to conduct ionic currents:

$\sigma=\operatorname{ASC} \times l=\rho^{-1}$

where ASC is the area specific conductance of membrane, which is equal to the multiplicative inverse of ASR, and $l$ is the ion-exchange membrane thickness. It is instructive to note that conductivity and resistivity are intensive properties, i.e., independent of membrane physical dimensions, whereas ASC and ASR are extensive properties. Introducing $\sigma$ and ASC enables the relationship between conductivity and permselectivity to be examined in an analytical framework akin to permeability-selectivity of gas separation and salt-rejecting membranes $[10,11]$.

\section{Model validation}

\subsection{Concentration and electric potential profile}

The boundary concentrations of counter- and co-ions are governed by chemical potential equilibrium and electroneutrality at the interface between the membrane and HC and LC solutions, Eqs. 3 and 8, respectively. At steady-state, ion fluxes $J_{\text {ct }}$ and $J_{\text {co }}$ are constant, and the concentration and electric potential profiles can then be numerically determined using the modified Nernst-Planck equation, Eq. (11), with the boundary condition concentrations. Fig. 2B shows representative $c_{\mathrm{ct}}, c_{\mathrm{co}}$, and $\varphi$ profiles across a CEM applied to reverse electrodialysis using $\mathrm{HC}$ and LC solutions of 600 and $17 \mathrm{mM} \mathrm{NaCl}$, respectively. Membrane properties are selected to simulate a typical commercial IEM: $c_{\text {fix }}=1.68 \mathrm{eq} / \mathrm{L}, f_{\mathrm{w}}=0.30, l=100 \mu \mathrm{m}$, and $\xi=1.08$ [30,50], with constant $\xi$ value being used throughout this study (unless specifically stated otherwise).

Under an external electric potential difference, $\Delta V^{\mathrm{m}}$, of $39.7 \mathrm{mV}$ between the solutions at the membrane interface, the counter- and coion fluxes are 3.75 and $0.0193 \mathrm{~mol} \mathrm{~m}^{-2} \mathrm{~h}^{-1}$, respectively (both in the direction of HC to LC side). Substituting $J_{\text {ct }}$ and $J_{\text {co }}$ into Eqs. (12) and (13) yields the concentration and electric potential gradients across the IEM. Within the membrane, the co-ion concentration, denoted by the red line, drops from 24.5 to $0.0351 \mathrm{mM}$ from the HC to LC solution interface ( $x=0$ and $100 \mu \mathrm{m}$, respectively), whereas counterion concentration decreases from 1.704 to $1.680 \mathrm{M}$ (blue line). Because of the charge neutrality constraint, local counterion concentration equals coion concentration plus fixed charge density of $1.68 \mathrm{eq} / \mathrm{L}$ (horizontal dashed green line).

The electric potential within the membrane, $\varphi^{\mathrm{m}}$, declines nonlinearly from the HC to LC interface, resulting in a net $\Delta \varphi^{\mathrm{m}}$ of $-44.1 \mathrm{mV}$ (orange line in Fig. 2B). This negative electric potential gradient across the CEM, along with negative concentration gradient, drives counterion (positive charges) transport from the HC to LC side (Eq. (11)), i.e., from left to right in Fig. 2A. For negatively-charged coions, the negative $d c^{\mathrm{m}} / d x$ overwhelms the electric potential migration term of the modified Nernst-Planck equation and, thus, co-ions also permeate from the HC to LC side, i.e., in the same direction as counterions. Because the counterion concentration within the membrane is over two orders of magnitude greater than $c_{\text {co }}$ due to charge exclusion, $J_{\mathrm{ct}}$ is also 194 fold higher than $J_{\mathrm{co}}$, thereby giving rise to the selective transport of counterions over co-ions. The net permeation of positive charges against $\Delta V^{\mathrm{m}}$ produces energy in RED.

In $\mathrm{ED}$, the slopes of the concentration profiles remain negative, while $\Delta \varphi^{\mathrm{m}}$ is switched to positive (modeling results not shown). The positive electric potential gradient is sufficiently large to overwhelm the negative counterion concentration gradient (first and second terms of Eq. (11), respectively). Hence, transport of counterions against $d c^{\mathrm{m}}$ / $d x$ is driven by the electric potential difference and $J_{\mathrm{ct}}$ is from the LC to HC side (as depicted in Fig. 2A). The co-ion flux is still from left to right and in the opposite direction as $J_{\text {ct }}$. Again, as in RED, $J_{\text {co }}$ is of drastically smaller magnitude compared to counterions because of charge exclusion by $c_{\text {fix }}$. Overall, the LC solution is, therefore, desalinated and the HC solution is concentrated. For both ED and RED, the selectivity for counterion transport by the IEM is always imperfect because co-ions cannot be completely excluded from the membrane matrix. Hence, current efficiency and permselectivity, Eqs. 15 and 16, are unavoidably $<1$.

\subsection{Counter- and co-ion fluxes in ED and RED}

Current density, $\hat{i}$, is ionic current normalized by membrane area and is calculated from the ion flux, $J$, using $\hat{i}_{i}=z_{i} F J_{i}$. Representative 
current densities of counterion, co-ion, and net fluxes as a function of external electric potential difference across the membrane, $\Delta V^{\mathrm{m}}$, are presented in Fig. 3 as solid blue, solid red, and dotted orange lines, respectively. The membrane and solutions properties are identical to the simulation analyzed in the preceding section (i.e., Fig. 2B): $c_{\text {fix }}$ $=1.68 \mathrm{eq} / \mathrm{L}, l=100 \mu \mathrm{m}, c^{\mathrm{s}, \mathrm{HC}}=600 \mathrm{mM} \mathrm{NaCl}$, and $c^{\mathrm{s}, \mathrm{LC}}=17 \mathrm{mM}$ $\mathrm{NaCl}$.

The regions of RED and ED operation can be discerned by the direction of the net ionic current density, $\hat{i}_{\text {tot }}$ : negative net currents signify ED desalination, whereas $\hat{i}_{\text {tot }}>0$ (and $\Delta V^{\mathrm{m}}>0$ ) indicates RED energy production. Because counter- and co-ions are oppositely charged, ionic current from co-ion flux opposes counterion current in RED (i.e., subtracts from net current) although $J_{\text {co }}$ and $J_{\text {ct }}$ are in the same direction; whereas in ED, co-ion current adds to the counterion current while $J_{\text {co }}$ and $J_{\mathrm{ct}}$ are opposing (Figs. 2A and 3). Open-circuit voltage (OCV), defined as $\Delta V^{\mathrm{m}}$ when net current is zero, delineates RED and ED operation regions on the I-V curve (Fig. 3) and is determined using an analytical approach described in a previous study [38]. For the CEM with high fixed charge density of $1.68 \mathrm{eq} / \mathrm{L}$ employed in this simulation, the OCV is $83.6 \mathrm{mV}$, slightly below the theoretical Nernst potential of $84.6 \mathrm{mV}$ between the different HC and LC concentrations of 600 and $17 \mathrm{mM}$ $\mathrm{NaCl}\left(\alpha_{\text {app }}=0.988\right)$. To desalinate the LC solution, $\Delta V^{\mathrm{m}}$ applied should overcome the OCV between two solutions. Conversely, to generate useful work from the controlled mixing between the LC and HC solutions, $0<\Delta V^{\mathrm{m}}<$ OCV.

In both RED and ED regions, the counterion current density show very good linear relationship with $\Delta V^{\mathrm{m}}$ (solid blue line in Fig. 3). For the range of $\Delta V^{\mathrm{m}}$ examined in Fig. 3, linear regression $R^{2}>0.9999$ is obtained. The highly linear dependence of $z_{\mathrm{ct}} F J_{\mathrm{ct}}$ on $\Delta V^{\mathrm{m}}$ is observed for IEMs with different properties and with different solution concentrations (details of simulation results not shown). Unlike counterion, the co-ion current density is clearly nonlinear (solid red line). But given that co-ion flux is only a small portion of the overall ion flux $(<2 \%)$, the effect on net current density is minimal and $\hat{i}_{\text {tot }}$ still behaves linearly with respect to $\Delta V^{\mathrm{m}}$ (dotted orange line). Even when the analysis simulates IEM permselectivity dropping significantly below 1 , the linear relationship between $\hat{i}_{\text {tot }}$ and $\Delta V^{\mathrm{m}}$ still holds (e.g., $R^{2}>0.99$ for $\alpha=0.50$ ). This modeling result provides theoretical justification for the common experimental approximation that IEMs behave as ohmic resistors in typical ED and RED operation.

\subsection{Validation of model predictions with empirical data}

The IEM transport model was validated using empirical data from literature $[16,33,51]$. Fig. 4 shows the simulated and experimental current density as a function of voltage for a 20-cell RED stack [16]. Literature values of the membrane properties of the commercial CEM and AEM installed in the RED stack were utilized in the model calculations $[33,51]$, while an empirical equation was employed to describe the conductivity-concentration relationship for $\mathrm{NaCl}$ solution [41]. Because the spacer shadow effect was not stated, two typical values of 0.65 and 0.8 were assumed (blue circle and violet triangle symbols, respectively) [52]. Summing the potential drop of the flow channels and IEMs across the RED stack yields the current density-voltage plot of Fig. 4. The simulation results are in good agreement with the empirical data (black square symbols), especially considering a comprehensive description of the experimental parameters was not available (e.g., system design and complete membrane characteristics), thus verifying the general accuracy of the model.

Fidelity of the transport model was further examined by individual membrane comparison. Using reported membrane structural properties, the model predicts the ionic conductivity of a commercial CEM in $1 \mathrm{M} \mathrm{NaCl}$ solution to be $7.18 \mathrm{mS} / \mathrm{cm}$, while direct current experiments measured $10.9 \mathrm{mS} / \mathrm{cm}$ [20]. Based solely on first principles and without additional fitting parameters, the model achieved reasonable accuracy. The discrepancy between modeling and experimental results could be attributed to either an incomplete description of the ion transport presented above, or experimental deficiencies in the measurement method. A recent study reports that the counterion condensation phenomenon as described by Manning's model potentially underestimates the observed diffusivity in IEMs [22]. Imprecisions can also creep into the simulation outcome from simplifying assumptions made in the transport model, such as the negligible water flux postulation. A possible shortcoming in experimental method that will have significant impact on the simulation output is neglect of the dry polymer free volume. The implicit assumption of volume additivity is commonly adopted when calculating the water volume fraction of hydrated charged polymers [53], but ignoring free volume in dry polymer [54] and volume contraction during water sorption [55] will lead to a nontrivial underestimation of $f_{\mathrm{w}}$. Because IEM conductivity is highly sensitive to the volume fraction of water (discussed later), a minute bias in $f_{\mathrm{w}}$ can create a sizable error in simulated conductivity. Furthermore, experimental measurements of IEM properties from different studies often yield widely disparate readings. For instance, for the same commercial CEM, Neosepta CMX, ionic conductivities of 2, 5 and $7.3 \mathrm{mS}$ / $\mathrm{cm}$ in $1 \mathrm{M} \mathrm{NaCl}$ electrolyte have been separately reported [20,56,57]. The inconsistency in measured values is likely due to differences in the experimental setup and difficulty in isolating the contribution of the diffusion layer resistance. Despite these limitations, the transport model presented here shows satisfactory reliability in estimating IEM performance parameters solely from intrinsic membrane properties.

\section{Influence of operating parameters on resistance and current efficiency}

Here, the effects of operating parameters, applied voltage and bulk solution concentrations, on membrane performance in ED and RED are examined. Simulated membrane properties of $c_{\mathrm{fix}}=1.68 \mathrm{eq} / \mathrm{L}, f_{\mathrm{w}}$ $=0.30, l=100 \mu \mathrm{m}$, and $\xi=1.08$ are held constant throughout this section.

\subsection{Electric potential across membrane affects current efficiency and permselectivity}

Current efficiency, CE (Eqs. (15a) and (15b)), and area specific

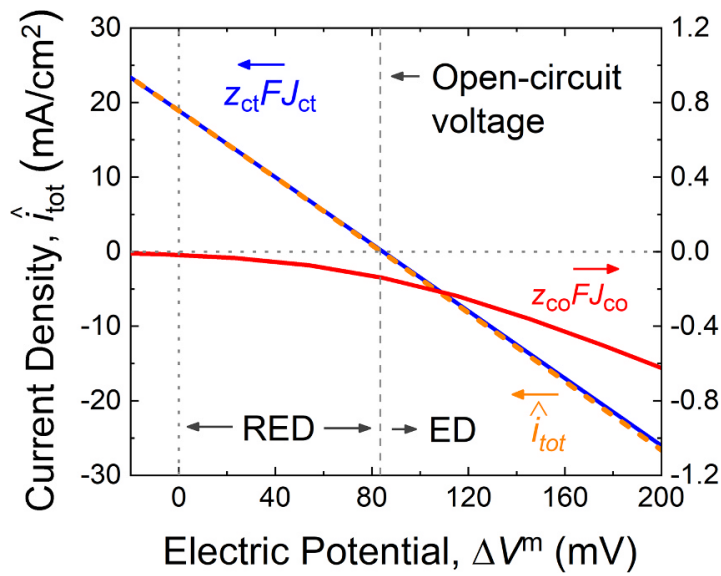

Fig. 3. Representative counterion and co-ion current densities, $\hat{i}=z F J$, as a function of external electric potential difference across the IEM, $\Delta V^{\mathrm{m}}$. The process is in reverse electrodialysis power generation for $\Delta V^{\mathrm{m}}$ between zero and the Nernst potential of the HC-LC solutions, while electrodialysis desalination occurs when $\Delta V^{\mathrm{m}}$ exceeds the Nernst potential. Note that undesired co-ion flux is in the same direction as counterion transport in RED (i.e., co- and counterion currents are in opposite directions) and is against counterion flux in ED (that is, both currents are in same direction). Simulation conditions: membrane properties $c_{\mathrm{fix}}=1.68 \mathrm{eq} / \mathrm{L}, f_{\mathrm{w}}=0.30$, and $l=100 \mu \mathrm{m}$; bulk solutions $c^{\mathrm{s}, \mathrm{HC}}$ $=600 \mathrm{mM} \mathrm{NaCl}$ and $c^{\mathrm{s}, \mathrm{LC}}=17 \mathrm{mM} \mathrm{NaCl}$. 


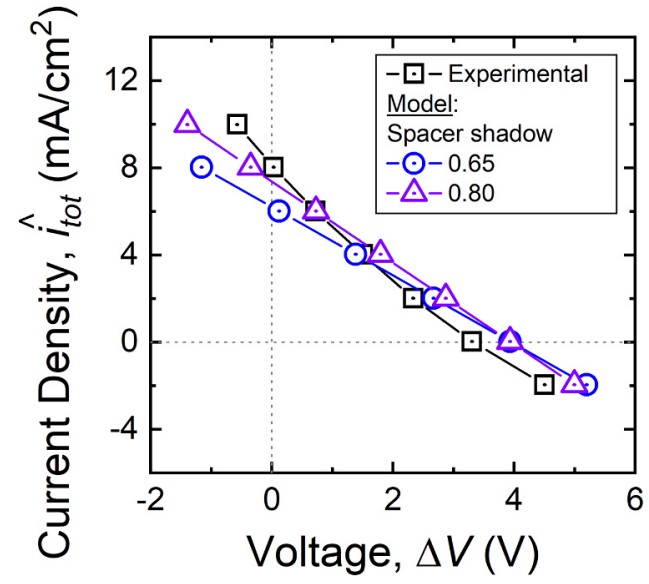

Fig. 4. Fitting of the model with literature data (black square symbols) [16], assuming spacer shadow effect of 0.65 and 0.80 (blue circle and violet triangle symbols, respectively). The model exhibited good agreement with the empirical data. Membrane properties were based on literature values [33,51]. The IEM stack with 20 cells were filled with 0.017 and $0.513 \mathrm{M} \mathrm{NaCl}$ as LC and HC feed streams, respectively, and all flow channels are $200 \mu \mathrm{m}$ thick.

resistance, ASR (Eq. (17)), as a function of $\Delta V^{\mathrm{m}}$ for ED and RED are shown in Fig. 5A and B, respectively. Concentration of the LC and HC solutions are 17 and $200 \mathrm{mM} \mathrm{NaCl}$ for ED to represent brackish water desalination to $1000 \mathrm{ppm}$ TDS product water, and 17 and $600 \mathrm{mM} \mathrm{NaCl}$ for RED to simulate salinity gradient power generation with seawaterriver water. The virtually constant ASR (red triangle symbols) further verifies that the effect of voltage on membrane resistance is negligible for both ED and RED, reinforcing earlier discussion on the effectively linear voltage-current relationship (Fig. 3) and is in agreement with reported empirical results of ohmic behavior of IEMs in under-limiting region [58].

While ASR remains practically constant, current efficiency and, equivalently, permselectivity are dependent on $\Delta V^{\mathrm{m}}$, especially as $\Delta V^{\mathrm{m}}$ $\rightarrow$ OCV (58.5 and $83.6 \mathrm{mV}$ for the ED and RED scenarios, respectively). Similar CE trends were exhibited for ED and RED, with symmetry about the OCV. Current efficiency dips drastically around the transition region between RED and ED, to around $90 \%$. As higher voltage is applied in ED desalination, $\mathrm{CE}$ increases but eventually levels off $(\approx 99.6 \%$ for the scenario simulated in Fig. 5A). This has been observed in a recent experimental study, where counterion transport number increased and plateaued as applied voltage rose [59]. RED power generation shows a similar trend to ED, but mirrored about the OCV (Fig. 5B). Current

\section{Internal Membrane Potential, $\Delta \varphi^{m}(\mathrm{mV})$}

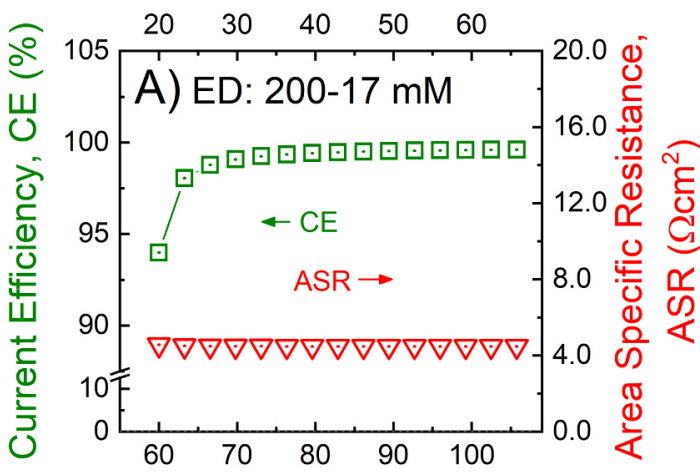

External Membrane Potential, $\Delta \mathrm{V}^{\mathrm{m}}(\mathrm{mV})$ efficiency reaches the highest at zero $\Delta V^{\mathrm{m}}(\approx 99.9 \%)$, but drops significantly as $\Delta V^{\mathrm{m}}$ approaches OCV. The decline in IEM selectivity around the OCV is due to the diminished driving force for counterion transport, Eq. (11), and is readily evident from Fig. 3: around the OCV, the electric potential difference within the membrane, $\Delta \varphi^{\mathrm{m}}$, is substantially lowered, resulting in reduced $J_{\text {ct }}$. Because, the magnitude of $J_{\text {co }}$ relative to $J_{\text {ct }}$ is now significantly elevated, CE and $\alpha$ are, therefore, compromised.

The dependence of CE and permselectivity on voltage has important implications for ED and RED operation. A previous analysis suggests that higher RED energy efficiencies can be obtained at lower current densities because internal resistance losses are suppressed, i.e., operate close to OCV [51]. However, this transport model indicates that permselectivity will be reduced, likely resulting in the overall energy efficiency being detrimentally affected. For accurate projections of RED performance, the voltage-dependent relationship of permselectivity should be included.

Membrane specifications provided by IEM manufacturers typically list the area specific resistance and permselectivity or, more precisely, the apparent permselectivity ( $\alpha_{\text {app }}=$ OCV divided by Nernst potential); such practice is also common in IEM studies $[15,16,60]$. However, both ASR and apparent permselectivity are not intrinsic membrane properties but, rather, dependent on the operating conditions. This analysis and also previous studies indicate ASR is effectively constant across the typical voltage range, but variation in permselectivity is significant and non-negligible. Hence, substantial error may be incurred if apparent permselectivity is used to approximate actual $\alpha$ during operation, because typical ED and RED processes utilize $\Delta V^{\mathrm{m}}$ significantly far away from the OCV. For the rest of the simulations in this study, $\Delta V^{\mathrm{m}}$ is $50 \%$ of OCV in RED to yield high power density [17], and set at 200\% OCV for ED to enable consistency in comparison.

\subsection{Influence of external solution concentrations}

Current efficiency and area specific resistance in RED are investigated for a range of bulk solution concentrations. Fig. $6 \mathrm{~A}$ shows $\mathrm{CE}$ and ASR (green square and red triangle symbols, respectively) as a function of $c^{\mathrm{s}, \mathrm{HC}}(0.6-3.0 \mathrm{M} \mathrm{NaCl}$, simulating salinity gradient power generation with seawater to hypersaline brines [9]). Concentration of $\mathrm{LC}$ solution is $17 \mathrm{mM} \mathrm{NaCl}$ to represent river water and $\Delta V^{\mathrm{m}}$ is $2 \times \mathrm{OCV}$ (as stated earlier).

\subsubsection{Increasing solution salinity compromises exclusion of co-ions}

As more saline HC solutions are employed in RED power generation, CE decreases from $99.4 \%$ to $95.9 \%$ (Fig. 6A). The reduced CE at higher

$$
\text { Internal Membrane Potential, } \Delta \varphi^{\mathrm{m}}(\mathrm{mV})
$$

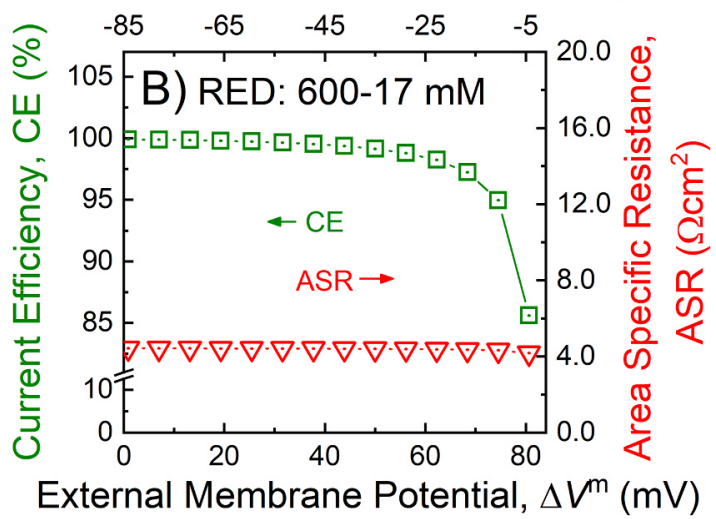

Fig. 5. Current efficiency, CE (Eqs. 15a and 15b), and area specific resistance, ASR (Eq. (17)), (green square, left vertical axis and red triangles, right vertical axis, respectively) as a function of external electric potential difference across the membrane, $\Delta V^{\mathrm{m}}$ for A) ED desalination of simulated brackish water at $200 \mathrm{mM}$ NaCl to produce product water of $1000 \mathrm{ppm}$ TDS $(=17 \mathrm{mM} \mathrm{NaCl}$ ) and B) RED power generation with seawater and river water (represented with 600 and $17 \mathrm{mM} \mathrm{NaCl}$, respectively). Membrane properties $c_{\mathrm{fix}}=1.68 \mathrm{eq} / \mathrm{L}, f_{\mathrm{w}}=0.30$, and $l=100 \mu \mathrm{m}$. 

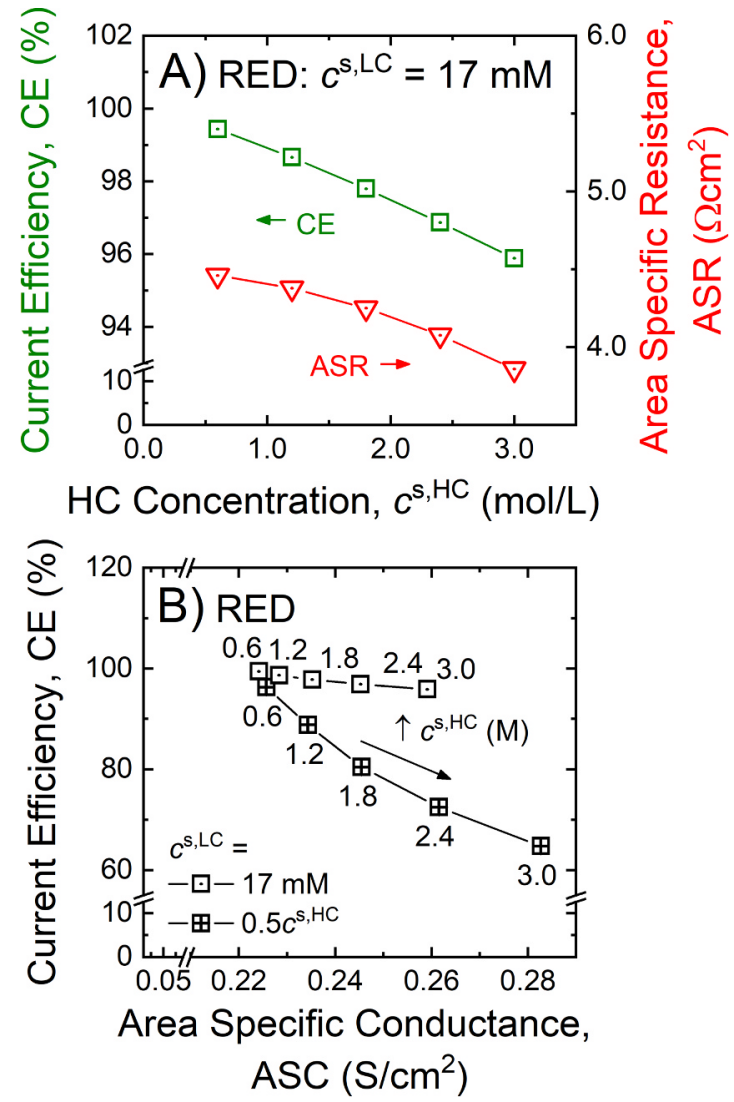

Fig. 6. A) Current efficiency, CE, and area specific resistance, ASR, (green square, left vertical axis and red triangles, right vertical axis, respectively) as a function of HC solution concentration for RED, with LC solution concentration of $17 \mathrm{mM} \mathrm{NaCl}$. B) Current efficiency and area specific conductance, ASC, (vertical and horizontal axes, respectively) with $c^{\mathrm{s}, \mathrm{HC}}$ increasing from $0.6-3.0 \mathrm{M}$ $\mathrm{NaCl}$ in RED (data point labels), representing utilization of seawater to hypersaline brine. The tradeoff relationship between CE and ASC is shown for constant $c^{\mathrm{s}, \mathrm{LC}}$ of $17 \mathrm{mM} \mathrm{NaCl}$ (to simulate river water) and varying LC solution concentration $=0.5 c^{\mathrm{s}, \mathrm{HC}}$ (dotted and crossed symbols, respectively). Membrane properties $c_{\mathrm{fix}}=1.68 \mathrm{eq} / \mathrm{L}, f_{\mathrm{w}}=0.30$, and $l=100 \mu \mathrm{m}$.

$c^{\mathrm{s}, \mathrm{HC}}$ signifies undesired leakage of co-ions across the IEM that gives rise to uncontrolled mixing and lowers extractable energy [17]. The deterioration of membrane selectivity is more pronounced when both the $\mathrm{HC}$ and LC solutions are of high salt concentration, as shown in Fig. 6B, where CE is plotted as a function of area specific conductance, ASC (multiplicative inverse of ASR). The data of Fig. 6A is replotted as dotted symbols in Fig. 6B, whereas crossed symbols denote HC-LC pairings $c^{\mathrm{s}, \mathrm{LC}}=1 / 2 c^{\mathrm{s}, \mathrm{HC}}$ (labels indicate HC solution concentration). The current efficiency decreases from $96.3 \%$ to $64.7 \%$ when the solution HC-LC concentrations increase from $0.6-0.3$ to $3.0-1.5 \mathrm{M} \mathrm{NaCl}$.

The depreciation of membrane selectivity in high external solution concentrations can be understood by examining the co-ion concentration within the IEM. At electrochemical potential equilibrium at the solution-membrane interface, the counter- and co-ion concentrations in the IEM can be determined by Eqs. 5-9. Blue circle and red square symbols of Fig. 7 denote $c_{\mathrm{ct}}^{\mathrm{m}}$ and $c_{\mathrm{co}}^{\mathrm{m}}$, respectively, at different bulk concentrations of external solution (note that all axes are on logarithmic scale). The fixed charge density within the membrane matrix, $c_{\mathrm{fix}}$, is represented by the horizontal dashed green line. Co-ion concentration increases as $c^{\mathrm{s}}$ is raised. To preserve charge balance, the equality $\left|z_{\mathrm{co}}\right| c_{\mathrm{co}}^{m}+c_{\mathrm{fix}}=\left|z_{\mathrm{ct}}\right| c_{\mathrm{ct}}^{m}$, Eq. (8), has to be maintained. Hence, $c_{\mathrm{ct}}^{\mathrm{m}}$ is also correspondingly increased, but because $c_{\mathrm{ct}}^{\mathrm{m}}$ is usually orders of magnitude greater than $c_{\mathrm{co}}^{\mathrm{m}}$, counterion concentration is practically equivalent to $c_{\text {fix }}$, except for $c^{\mathrm{s}}$ beyond $\approx c_{\mathrm{fix}}$ (vertical dotted line). The extent of IEM charge exclusion can be described by the concentration ratio of counter- to co-ions. The grey diamond symbols of Fig. 7 (right vertical axis) indicate $c_{\mathrm{ct}}^{\mathrm{m}} / c_{\mathrm{co}}^{\mathrm{m}}$ drops as bulk solution concentration increases. Therefore, the ability of the membrane fixed functional groups to exclude like-charged co-ions progressively diminishes with greater $c^{s}$ and is eventually overwhelmed when the external solution concentration is raised to around $c_{\mathrm{fix}}\left(c_{\mathrm{ct}}^{\mathrm{m}} / c_{\mathrm{co}}^{\mathrm{m}}<10\right.$ as $c^{\mathrm{s}} \approx c_{\mathrm{fix}}$, Fig. 7). In the lower concentration range, i.e., $<<c_{\text {fix }}$, changes to $c^{\mathrm{s}}$ and the resultant $c_{\mathrm{co}}^{\mathrm{m}}$ have practically negligible effect on IEM permselectivity as $c_{\mathrm{ct}}^{\mathrm{m}} / c_{\mathrm{co}}^{\mathrm{m}}$ is still adequately high.

In other words, effective IEM permselectivity is intrinsically confined to relatively low bulk solution concentrations. For RED power generation with river water and seawater, concentrations of the initial $\mathrm{HC}$ and LC feed streams are equivalent to about 600 and $17 \mathrm{mM} \mathrm{NaCl}$ [17]. These concentrations are lower than the common $c_{\text {fix }}(0.9-2.0 \mathrm{eq} /$ L) of current IEMs $[29,32]$. For the membrane simulated, counterion concentration is 185 times higher than co-ions, and therefore, current efficiency is high (99.4\%). Towards the end of controlled mixing in RED, $c^{\mathrm{s}, \mathrm{LC}}$ is considerably greater than initial and is represented by the crossed symbols in Fig. $6 \mathrm{~B}$, where $c^{\mathrm{s}, \mathrm{LC}}=1 / 2 c^{\mathrm{s}, \mathrm{HC}}$. For seawater-river water RED, CE is practically unaffected (96.3\%) when bulk concentration of the LC solution rises to as $300 \mathrm{mM} \mathrm{NaCl}$ as $c^{\mathrm{s}, \mathrm{LC}}$ is still below $c_{\text {fix }}=1.68 \mathrm{eq} / \mathrm{L}$. However, for RED power generation with feeds greater than seawater concentration, (e.g., hypersaline lakes and salt domes), the membrane permselectivity is severely compromised ( $\mathrm{CE}=$ $64.7 \%$ with $3.0-1.5 \mathrm{M} \mathrm{NaCl}$ ). This trend was empirically demonstrated in a previous study: when $c^{\mathrm{s}, \mathrm{HC}}$ is enlarged ten-fold from 0.5 to $5 \mathrm{M} \mathrm{NaCl}$ with $c^{\mathrm{s}, \mathrm{LC}}$ fixed at $0.1 \mathrm{M}$, permselectivity of the commercial membrane dropped from 0.90 to 0.77 [61]. As such, currently available IEMs are unlikely to be suitable for RED power generation with hypersaline feeds.

Parallel trends for current efficiency are seen in electrodialysis (Fig. 8). In brackish water ED desalination where the input feed stream is significantly lower than $35,000 \mathrm{ppm}$ TDS $(\approx 0.6 \mathrm{M} \mathrm{NaCl})$, the external solution concentrations are not sufficiently high to detrimentally affect co-ion exclusion (e.g., $\mathrm{CE}=99.8 \%$ for $100-50 \mathrm{mM} \mathrm{NaCl}$ ). Presently available IEMs, however, are not suitable for desalination of seawater or hypersaline feeds because of ineffectual selectivity. Similar to IEMs in RED, $c_{\mathrm{ct}}^{\mathrm{m}} / c_{\mathrm{co}}^{\mathrm{m}}$ drops drastically at such high salinities, seriously weakening co-ion exclusion and, thus, causing poor current efficiencies (e.g., $84.7 \%$ for the simulated membrane in $1.2-0.6 \mathrm{M} \mathrm{NaCl}$, representing desalination of seawater to brine twice as saline).

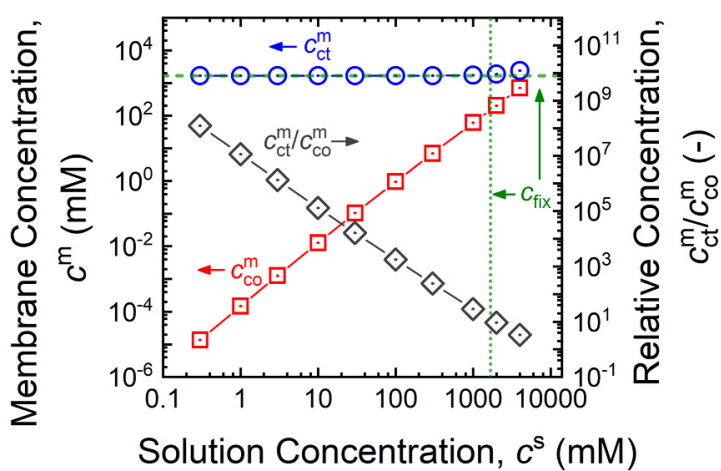

Fig. 7. Co-, counterion, and fixed charge concentrations in membrane (left vertical axis, red square symbols: $c_{\mathrm{co}}^{\mathrm{m}}$, blue circle symbols: $c_{\mathrm{ct}}^{\mathrm{m}}$, and horizontal dashed green line: $c_{\mathrm{fix}}^{\mathrm{m}}$, respectively) and ratio of counter- to co-ions, $c_{\mathrm{ct}}^{\mathrm{m}} / c_{\mathrm{co}}^{\mathrm{m}}$ (right vertical axis, grey diamond symbols), as a function of bulk solution concentration, $c^{\mathrm{s}}$. Membrane fixed charge density is also presented as vertical green dotted line to indicate the range of external solution concentrations beyond which co-ions exclusion is compromised. Note that all axes are on logarithmic scale. 


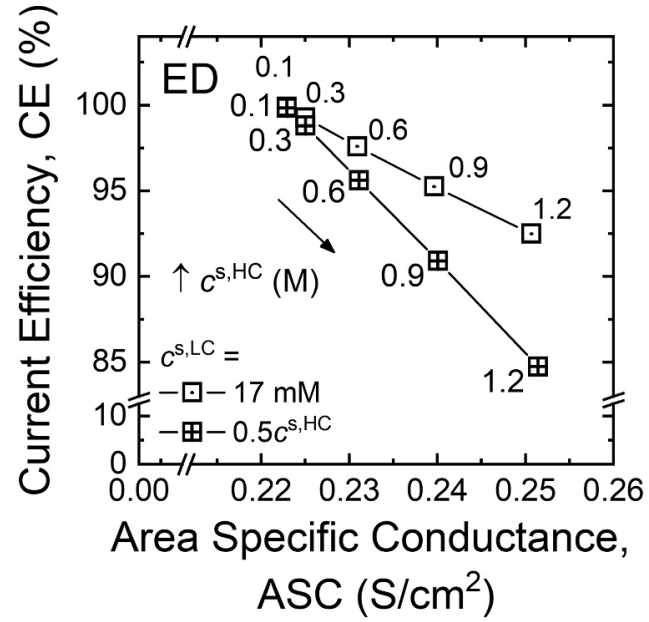

Fig. 8. Current efficiency and area specific conductance, ASC, (vertical and horizontal axes, respectively) with $c^{\mathrm{s}, \mathrm{HC}}$ increasing from 0.1 to $1.2 \mathrm{M} \mathrm{NaCl}$ in ED (data point labels), representing desalination of brackish water to brine of twice seawater concentration. The tradeoff relationship between CE and ASC is shown for constant $c^{\text {s,LC }}$ of $17 \mathrm{mM} \mathrm{NaCl}$ (simulate $1000 \mathrm{ppm}$ TDS product water) and varying LC solution concentration $=0.5 c^{\mathrm{s}, \mathrm{HC}}$ (dotted and crossed symbols, respectively). Membrane properties $c_{\mathrm{fix}}=1.68 \mathrm{eq} / \mathrm{L}, f_{\mathrm{w}}=0.30$, and $l=100 \mu \mathrm{m}$.

5.2.2. External concentrations cause tradeoff between conductivity and current efficiency

Whereas permselectivity is adversely affected by higher $c^{\mathrm{s}}$, membrane resistance is favorably lowered, although the improvement is modest (Figs. 6 and 8). For $c^{\mathrm{s}, \mathrm{LC}}=17 \mathrm{mM} \mathrm{NaCl}$, ASR declines from 4.46 to $3.86 \Omega \mathrm{cm}^{2}$ as $\mathrm{HC}$ concentration is elevated from $0.6 \mathrm{M}$ to $3.0 \mathrm{M} \mathrm{NaCl}$ in RED, and ASC reciprocally increases from 0.224 to $0.259 \mathrm{~S} / \mathrm{cm}^{2}$ (Fig. 6A and B). The same trend is observed in ED when LC concentration is $17 \mathrm{mM} \mathrm{NaCl}$ : ASC improves from 0.223 to $0.250 \mathrm{~S} / \mathrm{cm}^{2}$ for the simulate IEM by raising $c^{\mathrm{s}, \mathrm{HC}}$ from 0.1 to $1.2 \mathrm{M} \mathrm{NaCl}$ (Fig. 8). The marginal conductivity improvement is due to the greater ion concentration within the membrane matrix when $c^{\mathrm{s}}$ is high (Fig. 7). As described by the modified Nernst-Planck equation, Eq. (11), the elevated $c_{i}^{\mathrm{m}}$ consequently amplifies the ion fluxes, resulting in reduced resistance. The increased ionic current is carried by both counter- and co-ions, thereby accounting for the concomitant compromise in permselectivity. With greater LC solution concentrations, enhancements in membrane ionic conductivity are more pronounced, but still relatively small, e.g., for $c^{\mathrm{s}, \mathrm{HC}}=3.0 \mathrm{M} \mathrm{NaCl}$ in RED, ASC increases from 0.259 to $0.283 \mathrm{~S} / \mathrm{cm}^{2}$ as $c^{\mathrm{s}, \mathrm{LC}}$ rises from $17 \mathrm{mM}$ to $1.5 \mathrm{M}$ (Fig. 6B).

Thus, the operating condition of external solution concentrations produces a tradeoff between conductivity and current efficiency, as represented by the negative slopes of the ASC-CE trendlines in Figs. 6B and 8 , where increasing the bulk concentrations undesirably depresses membrane selectivity for counterions but slightly benefits conductivity. The tradeoff trend was consistently seen in simulations with membranes of different properties that are typical for current commercially available IEMs. This tradeoff has also been observed in recent experimental studies $[18,20,62]$. The concentration dependence of membrane conductivity was explained within the Nernst-Einstein framework for IEM in iso-concentration environment (i.e., $c^{\mathrm{s}, \mathrm{HC}}=c^{\mathrm{s}, \mathrm{LC}}$ and $d \mu_{i} / d x=$ 0 ) [20]. The transport model in this study further extends the theoretical rationale to encompass the more general scenario where there is a chemical potential gradient across the membrane that is representative of ED and RED operation.

The model presented here can be a useful tool to quantitatively approximate the tradeoff between permselectivity, $\alpha$, and conductivity, $\sigma$, at varying external concentrations. Simulated apparent permselectivity, $\alpha_{\text {app }}$, and $\sigma$ for different HC and LC solution concentrations are summarized in Fig. 9 for the representative IEM analyzed thus far $\left(c_{\mathrm{fix}}\right.$
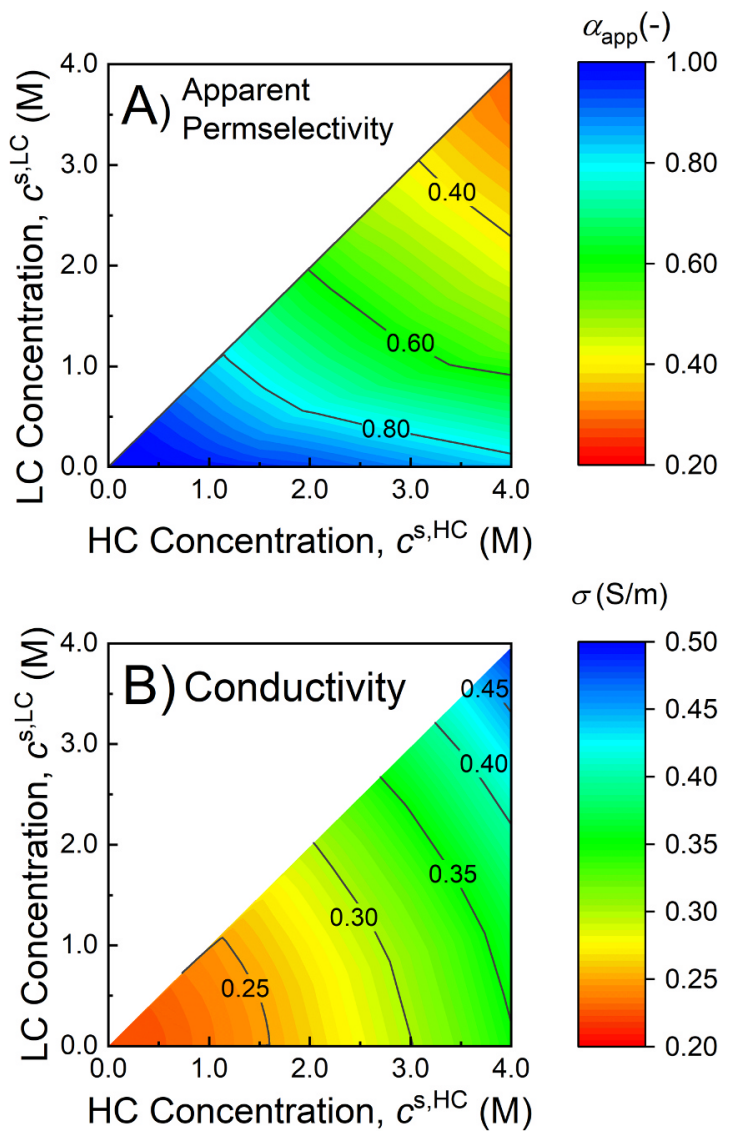

Fig. 9. Contour plot of A) apparent permselectivity and B) conductivity as a function of $\mathrm{HC}$ and LC bulk solution $\mathrm{NaCl}$ concentrations (horizontal and vertical axes, respectively). Apparent permselectivity is OCV divided by Nernst potential, whereas conductivity is averaged across current density of $-20-20 \mathrm{~mA} / \mathrm{cm}^{2}$. Membrane properties $c_{\mathrm{fix}}=1.68 \mathrm{eq} / \mathrm{L}, f_{\mathrm{w}}=0.30$, and $l$ $=100 \mu \mathrm{m}$.

$=1.68 \mathrm{eq} / \mathrm{L}, f_{\mathrm{w}}=0.30$, and $\left.l=100 \mu \mathrm{m}\right)$. Apparent permselectivity is commonly adopted for experimental characterization of IEMs [1,31] and is reported here to avoid the discrepancy arising from different applied voltages and operating modes. As discussed earlier, the membrane exhibits ohmic behavior across the range of ED and RED voltages and, therefore, conductivity for ED and RED is practically identical ( $\sigma$ reported in Fig. 9 is averaged across current density of -20 to $20 \mathrm{~mA} /$ $\mathrm{cm}^{2}$ ). The permselectivity data of Fig. 9 is in very good agreement with reported empirical results [61]. Limitations to the operating regime of ED and RED are clearly displayed: employing IEMs in salinities beyond seawater concentration significantly diminishes the permselectivity with only a marginal gain in conductivity, thus rendering RED inapt for power generation with hypersaline streams and confining ED to brackish water desalination.

\section{Conductivity-permselectivity relationships as a function of IEM properties}

Because ion-exchange capacity, swelling degree, thickness, fixed charge density, and water volume fraction of IEMs are intricately linked $[30,53]$, experimental approaches to investigate the impact of a single parameter on ED and RED are inevitably confounded by other properties that are simultaneously altered. On the other hand, the analytical framework employed in this study enables the influence of individual intrinsic membrane properties to be isolated for systematic examination. As such, the approach can more clearly elucidate the significance of the role played by the parameter, and inform rational customization 
of IEM properties for improved overall performance. Throughout this section, except for the parameter being inspected, all other IEM properties are held constant at IEC $=2.0 \mathrm{meq} / \mathrm{g}, \mathrm{SD}=0.36, l=100 \mu \mathrm{m}$, $\xi=1.08$, and polymer density, $\rho_{\mathrm{p}}=1.2 \mathrm{~g} / \mathrm{mL}$. The range of membrane structural properties investigated here are representative of common values reported in literature $[29,32,33]$. Unless stated otherwise, $c^{\mathrm{s}, \mathrm{HC}}$ $=200 \mathrm{mM} \mathrm{NaCl}$ and $c^{\mathrm{s}, \mathrm{LC}}=100 \mathrm{mM} \mathrm{NaCl}$ to simulate brackish water ED desalination, and RED uses $600-300 \mathrm{mM} \mathrm{NaCl}$ to represent seawater salinity gradient power generation.

\subsection{Impact of ion-exchange capacity}

\subsubsection{Co-ion exclusion is enhanced by raising ion-exchange capacity}

Ion-exchange capacity, IEC, is the number of fixed charges per unit weight of dry polymer [1]. The IEC of typical commercial ion-exchange membranes is in the range of $1-3 \mathrm{meq} / \mathrm{g}$ [30]. Because fixed charge density is normalized by the total volume of water-swollen membrane, $c_{\text {fix }}$ and IEC are related by the degree of hydration (which is characterized by the water volume fraction): $c_{\mathrm{fix}}=\left(1-f_{\mathrm{w}}\right)$ IEC. Fig. $10 \mathrm{~A}$ shows the equilibrium counter- and co-ion concentration ratio within the membrane as a function of external solution concentration, as described by Eqs. (5) and (9), for different fixed charge densities. The ratio of counter- to co-ions is related to fixed charge density by an approximately second-order power law for $c^{\mathrm{s}} \ll c_{\mathrm{fix}}$ : as $c_{\mathrm{fix}}$ increases sixfold from 0.5 to $3.0 \mathrm{eq} / \mathrm{L}, c_{\mathrm{ct}}^{\mathrm{m}} / c_{\mathrm{co}}^{\mathrm{m}}$ rises $\approx 36$ times (i.e., $c_{\mathrm{ct}}^{\mathrm{m}} / c_{\mathrm{co}}^{\mathrm{m}} \propto c_{\mathrm{fix}}{ }^{2}$ ). Thus, an IEM with higher $c_{\mathrm{fix}}$ is able to uphold a certain $c_{\mathrm{ct}}^{\mathrm{m}} / c_{\mathrm{co}}^{\mathrm{m}}$ in a more concentrated bulk solution, that is, maintain reasonable co-ion exclusion. Additionally, the counterion concentration is approximately equal to the fixed charge density to achieve charge balance. Hence, $c_{\mathrm{fix}}$ is critical for determining $c_{\mathrm{ct}}^{\mathrm{m}}$ and $c_{\mathrm{co}}^{\mathrm{m}}$ within the IEM.

\subsubsection{Increased charge density improves both conductivity and permselectivity}

The impact of IEC on conductivity and permselectivity is depicted in Fig. 10B. In the simulations, IEC is raised from 0.5 to $3.0 \mathrm{meq} / \mathrm{g}$. For RED, permselectivity increases from 0.586 to 0.967 and conductivity improves from 0.065 to $0.337 \mathrm{~S} / \mathrm{m}$; whereas in $\mathrm{ED}, \alpha$ is enhanced from 0.820 to 0.995 and $\sigma$ increases from 0.060 to $0.335 \mathrm{~S} / \mathrm{m}$. Increasing the ion-exchange capacity is simultaneously beneficial for conductivity and permselectivity, and influences both RED and ED operations. RED experiences greater $\alpha$ improvements as the $c_{\text {fix }}$ range is in the same order of magnitude as $c^{s}$. This trend is collaborated by experimental studies, as summarized in recent review articles [32,33].

The influence of IEC on $\alpha$ and $\sigma$ can be intuitively understood in the framework of chemical potential equilibrium and transport governed by the Nernst-Planck equation. With higher fixed charge density, exclusion of like-charged co-ions is enhanced (Fig. 10A), which is beneficial to the selective transport of counterions over co-ions. At the same time, to preserve electroneutrality, an increase in density of fixed charged groups raises the counterion concentration within the membrane matrix. Having more current carriers of mobile ions within the IEM yield greater ion fluxes that leads to better conductivity. Hence, increasing IEC is a direct method to simultaneously improve the key IEM performance parameters of permselectivity and ionic conductivity.

However, the approach of increasing IEC to attain more conductive and selective membranes is chemically and physically constrained in practice. Conventional IEMs have charged functional groups on the polymer matrix. Therefore, the functionalization chemistry imposes an upper limit on the achievable IEC. Additionally, when the concentration of fixed charge groups approaches within $\approx$ an order of magnitude of the ionization constant (i.e., $K_{\mathrm{a}}$ or $K_{\mathrm{b}}$ ), a significant fraction of the moieties will be unionized [63], hence, effectively lowering $c_{\text {fix }}$. Finally, intensifying IEC increases the polymer hydrophilicity that consequently raises the swelling degree of the IEM $[64,65]$. Membrane swelling due to water hydration dilutes $c_{\text {fix }}$ and, thus, opposes the $\alpha$ and $\sigma$ benefits of the enhanced IEC. Furthermore, greater water sorption exerts mounting osmotic swelling pressure on the polymer network. Beyond a certain point, the expansion stress exceeds the mechanical stability of the membrane and the polymer matrix ceases to form the required thin film $[66,67]$. These restrictions curtail the practically attainable fixed charge density for conventional IEMs.

\subsection{Influence of swelling degree}

6.2.1. Greater membrane hydration beneficially lowers tortuosity but reduces charge density

Swelling degree, SD, defined as the volume of water in the swollen IEM per unit polymer mass (i.e., dry membrane weight) [1], indicates the extent of membrane hydration. The volume fraction of water in the IEM, $f_{\mathrm{w}}$, is related to SD by

$f_{\mathrm{w}}=\frac{\mathrm{SD}}{\mathrm{SD}+\rho_{\mathrm{p}}^{-1}}$

where $\rho_{p}$ is the density of dry polymer. The water volume fraction, in turn, affects tortuosity, $\tau$, which describes the lengthened diffusional pathway across the water-swollen membrane, and can be approximated by considering ion transport is excluded from the stationary polymer chains $[45,46]$ :

$\tau=\frac{\left(2-f_{\mathrm{w}}\right)^{2}}{f_{\mathrm{w}}}$

The effective ion diffusivity ratio in water-swollen polymer to bulk aqueous phase is, thus, $D^{\mathrm{m}} / D^{\mathrm{s}}=f_{\mathrm{w}} / \tau$. Swelling reduces the tortuosity of ion-exchange polymer, which raises the ion mobility inside membrane.
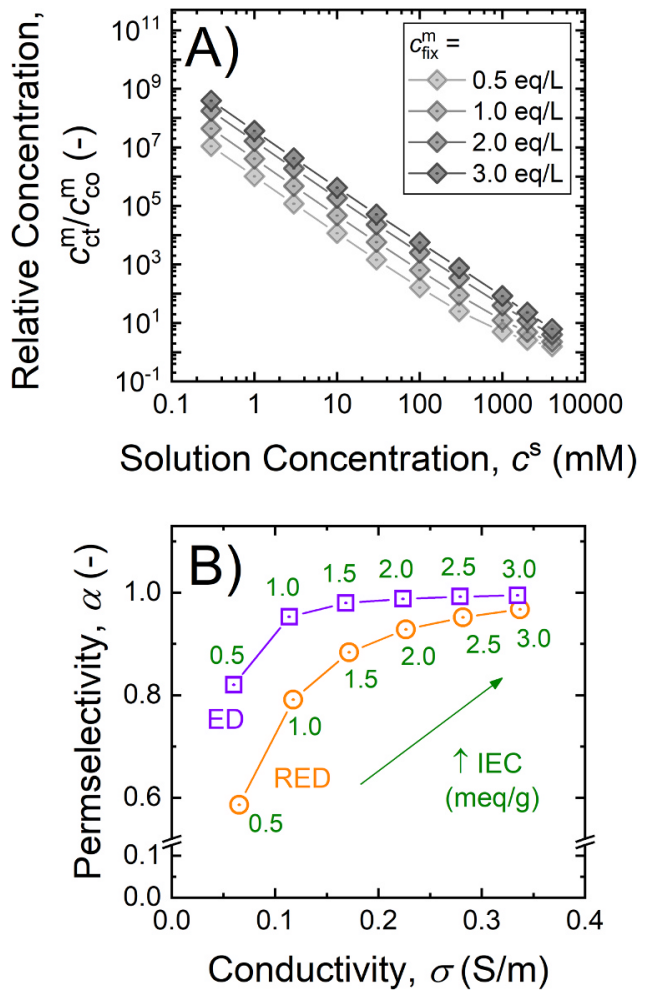

Fig. 10. A) Relative concentration of counter- to co-ions, $c_{\mathrm{ct}}^{\mathrm{m}} / c_{\mathrm{co}}^{\mathrm{m}}$, as a function of bulk solution concentration, $c^{\mathrm{s}}$, for different membrane fixed charge densities, $c_{\text {fix }}^{\mathrm{m}}$. Note that both axes are on logarithmic scale. B) Permselectivity, $\alpha$, and conductivity, $\sigma$, (vertical and horizontal axes, respectively) with increasing membrane ion-exchange capacity (indicated by green labels), for ED and RED (violet square and orange circle symbols, respectively). Membrane properties: $\mathrm{SD}=0.36 \mathrm{~cm}^{3} / \mathrm{g}, \rho_{\mathrm{p}}=1.2 \mathrm{~g} / \mathrm{mL}$, and $l=100 \mu \mathrm{m}$. Solution concentration employed are ED: $200-100 \mathrm{mM} \mathrm{NaCl}$ and RED: $600-300 \mathrm{mM} \mathrm{NaCl}$, to simulate brackish water desalination and seawater salinity gradient power generation, respectively. 
Fig. 11 A depicts $f_{\mathrm{w}}, \tau$, and $c_{\mathrm{fix}}$ as a function of SD, over the typical range for current IEMs [16]. As denoted by Eqs. (19) and (20), a larger SD augments the water volume fraction of the membrane matrix and reduces the tortuosity (blue triangle and violet diamond symbols, primary and secondary left vertical axes, respectively). For a given IEC (fixed at $2.0 \mathrm{meq} / \mathrm{g}$ in this simulation), $c_{\mathrm{fix}}$ is inversely proportional to SD: altering the swelling degree to higher levels dilutes $c_{\text {fix }}$ (green pentagon symbols, right vertical axis). As discussed earlier in Section 6.1 , the $c_{\mathrm{fix}}$ decline is unfavorable because the equilibrium $c_{\mathrm{ct}}^{\mathrm{m}}$ is lowered, which negatively influences conductivity. As such, increasing the IEM swelling degree is expected to produce opposing effects of reduced conductivity due to lowered fixed charge density, and enhanced ion transport because of increased water volume fraction and decreased diffusional path length.

\subsubsection{Altering swelling degree produces conductivity-permselectivity tradeoff}

Varying SD while keeping IEC and other membrane parameters constants produces a tradeoff between conductivity and permselectivity, as presented in Fig. 11B. When SD is raised from 0.15 to $1.15 \mathrm{~cm}^{3} / \mathrm{g}$ in the simulations, conductivity in ED increases from 0.059 to $0.744 \mathrm{~S} / \mathrm{m}$ while permselectivity drops from 0.997 to 0.813 ; for RED, the improvement in $\sigma$ from 0.059 to $0.855 \mathrm{~S} / \mathrm{m}$ is at the expense of $\alpha$, which declines from 0.986 to 0.635 . This tradeoff trend is reproducible across the range of typical membrane properties (results not shown), and is in agreement with reported observations of experimental studies $[5,15,16]$.

The effect of swelling degree on $\sigma$ and $\alpha$ can be explained with the IEM transport model presented in this study. As discussed earlier, when SD increases, membrane tortuosity drops and water volume fraction is augmented (Eqs. (19) and (20), and Fig. 11A), consequently boosting the effective ion diffusivities. According to the Nernst-Planck equation, Eq. (11), an increase in the diffusivity yields a proportional gain in ion flux that leads to enhanced conductivity. Although the lower $c_{\mathrm{fix}}$ due to a higher SD (Fig. 11A) has negative impact on conductivity (because of reduced ion concentration within the membrane matrix, see earlier discussion), the benefits to IEM structure outweigh the drawback and net $\sigma$ enhancement is attained. This trend of increased $\sigma$ with greater SD has been reported in a previous study, where 4 times increase of intrinsic conductivity was achieved when SD was raised from 0.167 to $0.295 \mathrm{~cm}^{3} / \mathrm{g}$. [64]. This empirical result matches very well with the simulation presented in Fig. 11B: raising SD from 0.15 to $0.35 \mathrm{~cm}^{3} / \mathrm{g}$ improved $\sigma \sim 3.7$ times. Further, the analysis indicates $>$ tenfold enhancement in conductivity can be attained by increasing membrane swelling degree from 0.15 to $1.15 \mathrm{~cm}^{3} / \mathrm{g}$.

The detrimental effect of greater SD on permselectivity can be rationalized by the dilution of $c_{\mathrm{fix}}$ (Fig. 11). A reduction in $c_{\mathrm{fix}}$ weakens the charge exclusion effect of the IEM, leading to a compromised $\alpha$ (Section 6.1). Empirical results of a recent study demonstrated that the permselectivity decreased when SD was lowered for the same IEC [65], corroborating the findings of this analysis. Due to the lower external solution concentrations, permselectivity for ED does not decrease as dramatically compared to RED (Fig. 11B).

Thus, membrane swelling degree has strong bearing on the key performance parameters of $\sigma$ and $\alpha$. Factors such as polymer structure, configuration, and crosslinking degree influences SD [53,68] and, hence, can be design levers to tune the balance between conductivity and permselectivity for customized IEM performance. Additionally, a higher SD would likely lead to a correspondingly larger membrane wet thickness that would yield a lower ASC, or equivalently, greater ASR. The analysis presented in Fig. 11B excludes the effect of $l$ by using thickness-normalized conductivity, $\sigma$. The influence of IEM thickness is examined in the next section.

\subsection{Effect of membrane thickness}

\subsubsection{Thinner membranes advantageously lower resistance}

The effects of IEM thickness, $l$, on permselectivity and area specific conductance, ASC ( $=\sigma / l$, Eq. (18)), are shown in Fig. 12. Because ASC and the reciprocal ASR are area-specific, i.e., membrane thickness effects are incorporated, they are of practical relevance in stack design for ED and RED processes. Assuming fixed ion-exchange capacity and swelling degree (IEC $=2.0 \mathrm{meq} / \mathrm{g}$ and $f_{\mathrm{w}}=0.30$ ) yields a constant conductivity that is independent of $l$. As the length of transport pathway scales with IEM thickness, ASR increases proportionally with membrane thickness. Conversely, ASC declines inversely from 0.373 to $0.112 \mathrm{~S}$ / $\mathrm{cm}^{2}$ when $l$ increases from 60 to $200 \mu \mathrm{m}$ (crossed symbol, right vertical axis of Fig. 12). The trends of thickness-independent conductivity and ASR $\propto l$ are supported by empirical results of previous experimental studies $[69,70]$. Furthermore, the simulated IEM exhibited effectively identical ASC in both ED and RED operations (that is, ohmic behavior is discussed in Section 5). Hence, thinner membranes advantageously enhance ASC for IEM applications.

\subsubsection{Permselectivity is independent of membrane thickness}

Crucially, the analysis shows that permselectivity is independent of membrane thickness. For the simulated $l$ range that is representative of current commercial IEMs $[30,31], \alpha$ is constant for both ED and RED modes (0.988 and 0.928 , respectively, Fig. 12$)$. The trend of preserved $\alpha$ with decreasing thickness is valid for the constant $\Delta V^{\mathrm{m}}$ applied in this analysis. However, operating thinner IEMs under constant current can result in lowered permselectivity due to $\Delta V^{\mathrm{m}}$ approaching the OCV with the improved conductivity (refer to discussion in Section 5.1), and as reported in a recent experimental study [71]. Reducing membrane thickness can produce lower resistance while preserving permselectivity, to improve the overall performance of IEM-based technologies. However, the technical limitations inherent to current IEM fabrication techniques, e.g., solvent evaporation casting, polymer blending and
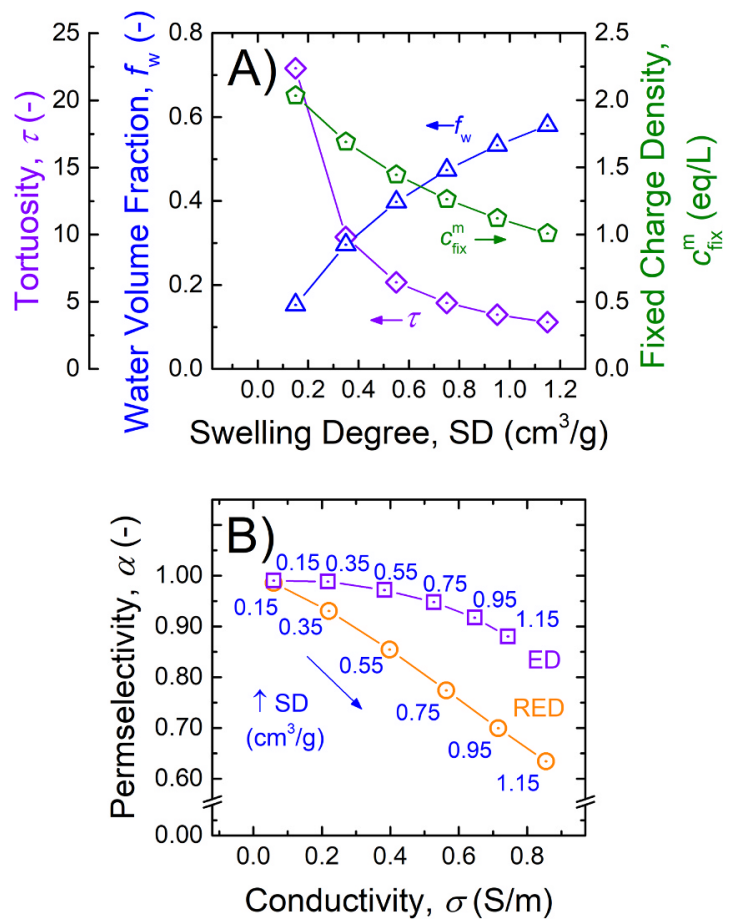

Fig. 11. A) Water volume fraction, $f_{\mathrm{w}}$, membrane tortuosity, $\tau$ (primary and secondary left vertical axes: blue triangle and violet diamond symbols, respectively), and fixed charge density, $c_{\mathrm{fix}}^{\mathrm{m}}$ (right vertical axis, green pentagon symbols), as a function of swelling degree, SD. B) Permselectivity, $\alpha$, and conductivity, $\sigma$, (vertical and horizontal axes, respectively) with increasing swelling degree (indicated by blue labels), for ED and RED (violet square and orange circle symbols, respectively). Membrane properties: IEC $=2.0 \mathrm{meq} / \mathrm{g}, \rho_{\mathrm{p}}$ $=1.2 \mathrm{~g} / \mathrm{mL}$, and $l=100 \mu \mathrm{m}$. ED employs $200-100 \mathrm{mM} \mathrm{NaCl}$ solutions, whereas RED uses 600-300 mM NaCl solutions. 


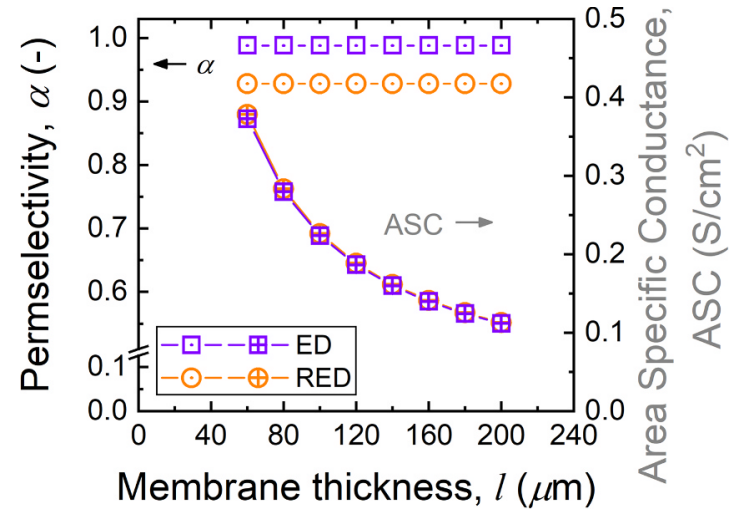

Fig. 12. Permselectivity, $\alpha$, and area specific conductance, ASC, (dotted symbols, left vertical axis and crossed symbols, right vertical axis, respectively) as a function of membrane thickness, $l$. Membrane properties: IEC $=2.0 \mathrm{meq} / \mathrm{g}, \mathrm{SD}$ $=0.36 \mathrm{~cm}^{3} / \mathrm{g}$, and $\rho_{\mathrm{p}}=1.2 \mathrm{~g} / \mathrm{mL}$. ED: $200-100 \mathrm{mM} \mathrm{NaCl}$ and RED: $600-300 \mathrm{mM} \mathrm{NaCl}$.

pore filling [33], together with the need for adequate mechanical robustness and defect-free films will constrain how thin the membranes can get. Hence, improvements in manufacturing methods and development of stronger materials are potential routes to robust ultrathin IEMs.

\section{Implications for ED desalination, RED energy production, and membrane development}

This study presents an IEM transport model that employs counterion condensation theory to analytically determine the experimentally inaccessible parameters of ion activity and diffusivity, and overcome a critical limitation hindering the application of the Nernst-Planck framework within the membrane matrix. The approach utilizes only intrinsic membrane properties, i.e., without additional fitting parameters, to determine process performance parameters that are in good agreement with reported measurements of experimental studies. While ionic conductivity, or equivalently, resistance, and permselectivity are commonly reported by membrane manufacturers and in literature as "IEM properties", this analysis shows that $\sigma$ and $\alpha$ are process-specific parameters that depend on operating conditions of applied voltage and bulk solution concentration, and are, hence, not truly inherent to the membrane. Instead, intrinsic membrane chemical and structural properties, such as ion-exchange capacity and swelling degree, are more useful process-independent parameters for benchmarking IEM performance.

The conductivity-permselectivity tradeoff observed in recent experimental studies are reproduced with the simulations here, and the fundamental mechanisms governing the interwoven relationship are laid out in the context of the Nernst-Planck framework: increasing the bulk solution concentration suppresses the charge-exclusion ability of the IEM, thereby reducing permselectivity but enhances conductivity due to greater ion concentration within the membrane matrix; whereas raising the swelling degree amplifies the effective ion diffusivity and improves conductivity, but concomitantly compromises permselectivity because the fixed charge density is diluted. Crucially, the analysis identifies the theoretical basis for the confined application of IEMs to sub-seawater salinities. Therefore, as is presently practiced, electrodialysis is only suitable for brackish water desalination. There has been recent interest in using hypersaline streams of saltworks brine and seawater desalination concentrate for reverse electrodialysis power generation [72-75]. However, the greatly diminished permselectivity at such hypersalinities would drastically deplete the energy extraction efficiency and render the process unfeasible.

Performance of ion-exchange membranes has advanced steadily over the years $[30,32,33]$ and the structure-property analysis of IEM transport presented here can further inform the rational development of customized membranes. Increasing the ion-exchange capacity should simultaneously enhance conductivity and permselectivity, but would also be accompanied greater IEM swelling as the more densely charged film sorbs larger solution volume. The elevated membrane swelling further improves conductivity but is at the expense of a lower permselectivity, as governed by the tradeoff relationship. Innovating fabrication techniques to drive the film thickness down while still maintaining adequate mechanical robustness offers the prospect of substantial gains in ionic conductivity without sacrificing permselectivity.

Further refinements can be made to the transport model presented here to sharpen the accuracy of the quantitative predictions and broaden the potential applications. For instance, accounting for water flux across the membrane will yield a more complete description of IEM transport $[76,77]$. The selectivity between different counterions (or coions) [78] can also be further studied through this model by simulating multiple ions. The Nernst-Planck framework employed here can be extended to incorporate concentration polarization at the solutionmembrane interface, a practically salient phenomenon that was not included in the scope of this study [79,80]. Eventually, integrating the one-dimension transport simulation into a multiscale model will enable the overall performance of system-level ED and RED stacks to be more precisely projected.

\section{Acknowledgements}

We acknowledge financial support from the United States Bureau of Reclamation, Grant R16AC00124. In addition, we thank Dr. Alan C. West and Jonathan D. Vardner for the helpful discussions.

\section{References}

[1] H. Strathmann, Ion-Exchange Membrane Separation Processes, Elsevier, 2004.

[2] H. Strathmann, Electrodialysis, a mature technology with a multitude of new applications, Desalination 264 (2010) 268-288.

[3] S. Bose, T. Kuila, T.X.H. Nguyen, N.H. Kim, K.-t. Lau, J.H. Lee, Polymer membranes for high temperature proton exchange membrane fuel cell: recent advances and challenges, Prog. Polym. Sci. 36 (2011) 813-843.

[4] G. Merle, M. Wessling, K. Nijmeijer, Anion exchange membranes for alkaline fuel cells: a review, J. Membr. Sci. 377 (2011) 1-35.

[5] P. Długołęcki, K. Nymeijer, S. Metz, M. Wessling, Current status of ion exchange membranes for power generation from salinity gradients, J. Membr. Sci. 319 (2008) $214-222$.

[6] H. Strathmann, A. Grabowski, G. Eigenberger, Ion-exchange membranes in the chemical process industry, Ind. Eng. Chem. Res. 52 (2013) 10364-10379.

[7] T.W. Xu, C.H. Huang, Electrodialysis-based separation technologies: a critical review, AIChE J. 54 (2008) 3147-3159.

[8] J.W. Post, H.V. Hamelers, C.J. Buisman, Energy recovery from controlled mixing salt and fresh water with a reverse electrodialysis system, Environ. Sci. Technol. 42 (2008) 5785-5790.

[9] N.Y. Yip, D. Brogioli, H.V.M. Hamelers, K. Nijmeijer, Salinity gradients for sustainable energy: primer, progress, and prospects, Environ. Sci. Technol. 50 (2016) 12072-12094.

[10] H.B. Park, J. Kamcev, L.M. Robeson, M. Elimelech, B.D. Freeman, Maximizing the right stuff: the trade-off between membrane permeability and selectivity, Science 356 (2017).

[11] B.D. Freeman, Basis of permeability/selectivity tradeoff relations in polymeric gas separation membranes, Macromolecules 32 (1999) 375-380.

[12] G.M. Geise, H.B. Park, A.C. Sagle, B.D. Freeman, J.E. McGrath, Water permeability and water/salt selectivity tradeoff in polymers for desalination, J. Membr. Sci. 369 (2011) 130-138.

[13] A. Mehta, A.L. Zydney, Permeability and selectivity analysis for ultrafiltration membranes, J. Membr. Sci. 249 (2005) 245-249.

[14] N.Y. Yip, M. Elimelech, Performance limiting effects in power generation from salinity gradients by pressure retarded osmosis, Environ. Sci. Technol. 45 (2011) 10273-10282.

[15] G.M. Geise, M.A. Hickner, B.E. Logan, Ionic resistance and permselectivity tradeoffs in anion exchange membranes, ACS Appl. Mater. Interface 5 (2013) 10294-10301.

[16] E. Guler, R. Elizen, D.A. Vermaas, M. Saakes, K. Nijmeijer, Performance-determining membrane properties in reverse electrodialysis, J. Membr. Sci. 446 (2013) 266-276.

[17] N.Y. Yip, D.A. Vermaas, K. Nijmeijer, M. Elimelech, Thermodynamic, energy efficiency, and power density analysis of reverse electrodialysis power generation with natural salinity gradients, Environ. Sci. Technol. 48 (2014) 4925-4936.

[18] A.H. Galama, D.A. Vermaas, J. Veerman, M. Saakes, H.H.M. Rijnaarts, J.W. Post, 
K. Nijmeijer, Membrane resistance: the effect of salinity gradients over a cation exchange membrane, J. Membr. Sci. 467 (2014) 279-291.

[19] N. Berezina, N. Kononenko, O. Dyomina, N. Gnusin, Characterization of ion-exchange membrane materials: properties vs structure, Adv. Colloid Interface Sci. 139 (2008) 3-28.

[20] J. Kamcev, R. Sujanani, E.S. Jang, N. Yan, N. Moe, D.R. Paul, B.D. Freeman, Salt concentration dependence of ionic conductivity in ion exchange membranes, J. Membr. Sci. 547 (2018) 123-133.

[21] B.P. Zhang, J.G. Hong, S.H. Xie, S.M. Xia, Y.S. Chen, An integrative modeling and experimental study on the ionic resistance of ion-exchange membranes, J. Membr. Sci. 524 (2017) 362-369.

[22] J. Kamcev, D.R. Paul, G.S. Manning, B.D. Freeman, Ion diffusion coefficients in ion exchange membranes: significance of counterion condensation, Macromolecules 51 (2018) 5519-5529.

[23] L.M. Robeson, H.H. Hwu, J.E. McGrath, Upper bound relationship for proton exchange membranes: empirical relationship and relevance of phase separated blends, J. Membr. Sci. 302 (2007) 70-77.

[24] K. Kontturi, L. Murtomäki, J.A. Manzanares, Ionic Transport Processes: In Electrochemistry and Membrane Science, Oxford University Press, 2008.

[25] A. Campione, L. Gurreri, M. Ciofalo, G. Micale, A. Tamburini, A. Cipollina, Electrodialysis for water desalination: a critical assessment of recent developments on process fundamentals, models and applications, Desalination (2018).

[26] M. Tedesco, H.V.M. Hamelers, P.M. Biesheuvel, Nernst-Planck transport theory for (reverse) electrodialysis: I. Effect of co-ion transport through the membranes, J. Membr. Sci. 510 (2016) 370-381.

[27] A. Moya, A Nernst-Planck analysis on the contributions of the ionic transport in permeable ion-exchange membranes to the open circuit voltage and the membrane resistance in reverse electrodialysis stacks, Electrochim. Acta 238 (2017) 134-141.

[28] J. Kamcev, M. Galizia, F.M. Benedetti, E.S. Jang, D.R. Paul, B.D. Freeman, G.S. Manning, Partitioning of mobile ions between ion exchange polymers and aqueous salt solutions: importance of counter-ion condensation, Phys. Chem. Chem. Phys. 18 (2016) 6021-6031.

[29] J. Kamcev, D.R. Paul, G.S. Manning, B.D. Freeman, Predicting salt permeability coefficients in highly swollen, highly charged ion exchange membranes, ACS Appl. Mater. Interface 9 (2017) 4044-4056.

[30] T. Xu, Ion exchange membranes: state of their development and perspective, J. Membr. Sci. 263 (2005) 1-29.

[31] T. Sata, Ion exchange membranes: preparation, characterization, modification and application, Royal Society of chemistry, 2007.

[32] J.G. Hong, B.P. Zhang, S. Glabman, N. Uzal, X.M. Dou, H.G. Zhang, X.Z. Wei, Y.S. Chen, Potential ion exchange membranes and system performance in reverse electrodialysis for power generation: a review, J. Membr. Sci. 486 (2015) 71-88.

[33] J. Ran, L. Wu, Y.B. He, Z.J. Yang, Y.M. Wang, C.X. Jiang, L. Ge, E. Bakangura, T.W. Xu, Ion exchange membranes: new developments and applications, J. Membr. Sci. 522 (2017) 267-291.

[34] M. Turek, B. Bandura, Renewable energy by reverse electrodialysis, Desalination 205 (2007) 67-74.

[35] A.J. Bard, L.R. Faulkner, J. Leddy, C.G. Zoski, Electrochemical Methods: Fundamentals and Applications, wiley, New York, 1980.

[36] H.B. Callen, Thermodynamics and an Introduction to Thermostatistics, Wiley, 1985.

[37] F.G. Donnan, Theorie der Membrangleichgewichte und Membranpotentiale bei Vorhandensein von nicht dialysierenden Elektrolyten. Ein Beitrag zur physikalisch-chemischen Physiologie, Ber. Bunsenges. Phys. Chem. 17 (1911) 572-581.

[38] A. Galama, J. Post, H. Hamelers, V. Nikonenko, P. Biesheuvel, On the origin of the membrane potential arising across densely charged ion exchange membranes: How well does the Teorell-Meyer-Sievers theory work? J. Membr. Sci. Res. 2 (2016) $128-140$.

[39] G.S. Manning, Limiting laws and counterion condensation in polyelectrolyte solutions II. Self-diffusion of the small ions, J. Chem. Phys. 51 (1969) 934-938.

[40] J. Kamcev, D.R. Paul, B.D. Freeman, Effect of fixed charge group concentration on equilibrium ion sorption in ion exchange membranes, J. Mater. Chem. A 5 (2017) 4638-4650.

[41] R.A. Robinson, R.H. Stokes, Electrolyte Solutions, Second edition, Butterworths Scientific Publications, London, 1959.

[42] K.S. Pitzer, Thermodynamics of electrolytes. I. Theoretical basis and general equations, J. Phys. Chem. 77 (1973) 268-277.

[43] K.S. Pitzer, G. Mayorga, Thermodynamics of electrolytes. II. activity and osmotic coefficients for strong electrolytes with one or both ions univalent, J. Phys. Chem. 77 (1973) 2300-2308.

[44] F.G. Helfferich, Ion Exchange, Courier Corporation, 1962.

[45] J.S. Mackie, P. Meares, The diffusion of electrolytes in a cation-exchange resin membrane. 1 Theoretical, Proc. R. Soc. Lond. Ser.-A 232 (1955) 498-509.

[46] J.S. Mackie, P. Meares, The diffusion of electrolytes in a cation-exchange resin membrane .2. Experimental, Proc. R. Soc. Lond. Ser.-A 232 (1955) 510-518.

[47] J.G. Wijmans, R.W. Baker, The solution-diffusion model: a review, J. Membr. Sci. 107 (1995) 1-21.

[48] D.R. Paul, Reformulation of the solution-diffusion theory of reverse osmosis, J. Membr. Sci. 241 (2004) 371-386.

[49] N. Rosenberg, C. Tirrell, Limiting currents in membrane cells, Ind. Eng. Chem. 49 (1957) 780-784.

[50] J. Kamcev, Ion sorption and transport in ion exchange membranes: importance of counter-ion condensation, in, 2016.

[51] J. Veerman, R.M. de Jong, M. Saakes, S.J. Metz, G.J. Harmsen, Reverse electrodialysis: comparison of six commercial membrane pairs on the thermodynamic efficiency and power density, J. Membr. Sci. 343 (2009) 7-15.

[52] P. Długołęcki, J. Dąbrowska, K. Nijmeijer, M. Wessling, Ion conductive spacers for increased power generation in reverse electrodialysis, J. Membr. Sci. 347 (2010) 101-107.

[53] G.M. Geise, D.R. Paul, B.D. Freeman, Fundamental water and salt transport properties of polymeric materials, Prog. Polym. Sci. 39 (2014) 1-42.

[54] R.P. White, J.E. Lipson, Polymer free volume and its connection to the glass transition, Macromolecules 49 (2016) 3987-4007.

[55] D. Nandan, H. Mohan, R. Iyer, Methanol and water uptake, densities, equivalental volumes and thicknesses of several uni-and divalent ionic perfluorosulphonate exchange membranes (Nafion-117) and their methanol-water fractionation behaviour at 298 K, J. Membr. Sci. 71 (1992) 69-80.

[56] A.H. Galama, N.A. Hoog, D.R. Yntema, Method for determining ion exchange membrane resistance for electrodialysis systems, Desalination 380 (2016) 1-11.

[57] N. Pismenskaya, E. Belova, V. Nikonenko, C. Larchet, Electrical conductivity of cation-and anion-exchange membranes in ampholyte solutions, Russ. J. Electrochem. 44 (2008) 1285-1291.

[58] J. Balster, M.H. Yildirim, D.F. Stamatialis, R. Ibanez, R.G.H. Lammertink, V. Jordan, M. Wessling, Morphology and microtopology of cation-exchange polymers and the origin of the overlimiting current, J. Phys. Chem. B 111 (2007) 2152-2165.

[59] J.T. Vardner, T. Ling, S.T. Russell, A.M. Perakis, Y. He, N.W. Brady, S.K. Kumar, A.C. West, Method of measuring salt transference numbers in ion-selective membranes, J. Electrochem. Soc. 164 (2017) A2940-A2947.

[60] E. Guler, Y. Zhang, M. Saakes, K. Nijmeijer, Tailor-made anion-exchange membranes for salinity gradient power generation using reverse electrodialysis, ChemSusChem 5 (2012) 2262-2270.

[61] A. Daniilidis, D.A. Vermaas, R. Herber, K. Nijmeijer, Experimentally obtainable energy from mixing river water, seawater or brines with reverse electrodialysis, Renew. Energ. 64 (2014) 123-131.

[62] G.M. Geise, A.J. Curtis, M.C. Hatzell, M.A. Hickner, B.E. Logan, Salt concentration differences alter membrane resistance in reverse electrodialysis stacks, Environ. Sci. Technol. Lett. 1 (2014) 36-39.

[63] S. Takamuku, A. Wohlfarth, A. Manhart, P. Rader, P. Jannasch, Hypersulfonated polyelectrolytes: preparation, stability and conductivity, Polym. Chem. 6 (2015) 1267-1274.

[64] W. Cui, J. Kerres, G. Eigenberger, Development and characterization of ion-exchange polymer blend membranes, Sep. Purif. Technol. 14 (1998) 145-154.

[65] D.H. Cho, K.H. Lee, Y.M. Kim, S.H. Park, W.H. Lee, S.M. Lee, Y.M. Lee, Effect of cationic groups in poly(arylene ether sulfone) membranes on reverse electrodialysis performance, Chem. Commun. 53 (2017) 2323-2326.

[66] G. He, Z. Li, J. Zhao, S. Wang, H. Wu, M.D. Guiver, Z. Jiang, Nanostructured ion-exchange membranes for fuel cells: recent advances and perspectives, Adv. Mater 27 (2015) 5280-5295.

[67] M. Kariduraganavar, R. Nagarale, A. Kittur, S. Kulkarni, Ion-exchange membranes: preparative methods for electrodialysis and fuel cell applications, Desalination 197 (2006) 225-246.

[68] R. Toomey, D. Freidank, J. Ruhe, Swelling behavior of thin, surface-attached polymer networks, Macromolecules 37 (2004) 882-887.

[69] G.G. Wang, Y.M. Weng, D. Chu, R.R. Chen, D. Xie, Developing a polysulfone-based alkaline anion exchange membrane for improved ionic conductivity, J. Membr. Sci. 332 (2009) 63-68.

[70] G.J. Hwang, H. Ohya, T. Nagai, Ion exchange membrane based on block copolymers. Part III: preparation of cation exchange membrane, J. Membr. Sci. 156 (1999) $61-65$.

[71] M. Tedesco, H.V.M. Hamelers, P.M. Biesheuvel, Nernst-Planck transport theory for (reverse) electrodialysis: .III. Optimal membrane thickness for enhanced process performance, J. Membr. Sci. 565 (2018) 480-487.

[72] M. Tedesco, E. Brauns, A. Cipollina, G. Micale, P. Modica, G. Russo, J. Helsen, Reverse electrodialysis with saline waters and concentrated brines: a laboratory investigation towards technology scale-up, J. Membr. Sci. 492 (2015) 9-20.

[73] M. Tedesco, A. Cipollina, A. Tamburini, G. Micale, J. Helsen, M. Papapetrou, REAPower: use of desalination brine for power production through reverse electrodialysis, Desalin. Water Treat. 53 (2015) 3161-3169.

[74] M. Tedesco, C. Scalici, D. Vaccari, A. Cipollina, A. Tamburini, G. Micale, Performance of the first reverse electrodialysis pilot plant for power production from saline waters and concentrated brines, J. Membr. Sci. 500 (2016) 33-45.

[75] M. Tedesco, A. Cipollina, A. Tamburini, G. Micale, Towards $1 \mathrm{~kW}$ power production in a reverse electrodialysis pilot plant with saline waters and concentrated brines, $\mathrm{J}$. Membr. Sci. 522 (2017) 226-236.

[76] M. Tedesco, H.V.M. Hamelers, P.M. Biesheuvel, Nernst-Planck transport theory for (reverse) electrodialysis: II. Effect of water transport through ion-exchange membranes, J. Membr. Sci. 531 (2017) 172-182.

[77] A. Zlotorowicz, R.V. Strand, O.S. Burheim, O. Wilhelmsen, S. Kjelstrup, The permselectivity and water transference number of ion exchange membranes in reverse electrodialysis, J. Membr. Sci. 523 (2017) 402-408.

[78] T. Luo, S. Abdu, M. Wessling, Selectivity of ion exchange membranes: a review, J. Membr. Sci. (2018).

[79] P. Dlugolecki, B. Anet, S.J. Metz, K. Nijmeijer, M. Wessling, Transport limitations in ion exchange membranes at low salt concentrations, J. Membr. Sci. 346 (2010) $163-171$.

[80] J.J. Krol, M. Wessling, H. Strathmann, Concentration polarization with monopolar ion exchange membranes: current-voltage curves and water dissociation, J. Membr. Sci. 162 (1999) 145-154. 